\title{
Minimum Energy Pulse Synthesis via the Inverse Scattering Transform
}

\author{
Charles L. Epstein* \\ LSNI, Department of Radiology, HUP \\ and \\ Department of Mathematics, University of Pennsylvania
}

Version 2.1 March 7, 2003

\begin{abstract}
This paper considers a variety of problems in the design of selective RF-pulses. We apply a formula of Zakharov and Manakov to directly relate the energy of an RF-envelope to the magnetization profile and certain auxiliary parameters used in the inverse scattering transform (IST) approach to RF-pulse synthesis. This allows a determination of the minimum possible energy for a given magnetization profile. We give an algorithm to construct the minimum energy RF-envelope which includes an algorithm for solving the Gel'fand-Levitan-Marchenko equations. The SLR method is shown to be a special (singular) case of the IST approach. We analyze the approximations used in the SLR approach. The minimum energy RFenvelope is computed for a variety of examples.
\end{abstract}

\section{Contents}

$\begin{array}{lll}\text { 0 } & \text { Introduction } & 2\end{array}$

1 The Bloch Equation 4

2 Scattering Theory for the ZS-System 6

3 Inverse Scattering for the ZS-System 11

4 The Energy of the RF-Envelope 14

* Research partially supported by NSF grants DMS99-70487 and DMS02-07123. Address: Department of Mathematics, University of Pennsylvania, Philadelphia, PA. E-mail: cle@math.upenn.edu

Keywords: nuclear magnetic resonance, imaging, RF-pulse synthesis, selective excitation, inverse scattering, Marchenko equation, minimum energy. 
5 Solving the Marchenko Equation 16

6 What Is Time?

7 Analysis of the Hard Pulse Approximation 22

8 Examples 28

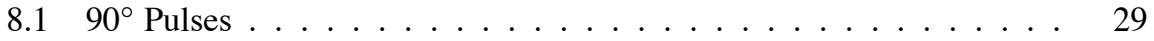

8.2 Two band pulses . . . . . . . . . . . . . . . . . . . . . 32

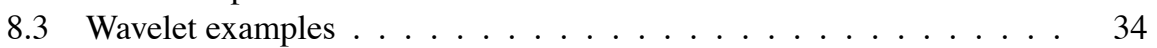

8.4 Power computations . . . . . . . . . . . . . . . 35

9 Conclusion $\quad 35$

10 Appendix: Mathematical Proofs 36

\section{Introduction}

The design of RF pulse sequences to produce selective excitation is a problem of central importance in all applications of nuclear magnetic resonance. The oldest method of pulse synthesis is the Fourier transform method. Notwithstanding the fact that this method is an approximation for all nonzero flip angles, it gives usable results for flip angles up to about $\frac{\pi}{2}$. Over the past fifteen years two, more exact approaches to solving this problem have been introduced and explored. The slightly older, generally accepted method is the Shinnar-Le Roux or SLR algorithm introduced independently by M. Shinnar and his co-workers and P. Le Roux. See for example [17], [18], or [10]. A more complete list of the original papers can be found in the references to [13]. The second method is to use the inverse scattering transform or IST which was first introduced as a technique for pulse synthesis in [9] and later refined in [14, 5, 6].

From the practical perspective, either method of pulse synthesis is rather demanding and requires considerable computation. From the theoretical perspective, the problem of pulse synthesis is very easy. Indeed, it is clear from the inverse scattering approach that there is an infinite dimensional space of RF-envelopes that will produce any reasonable magnetization profile. The "real" problem of pulse synthesis is therefore to find an "optimal" RF-envelope to produce a given magnetization profile. In this paper we explore the IST approach and show how it can be used to design RF-envelopes which utilize the minimum possible energy, for a given magnetization profile. At the center of our discussion is a formula expressing the energy in the RF-envelope explicitly in terms of the magnetization profile and certain free parameters that arise in the IST method. Formulæ of this type were discovered, in the context of inverse scattering for the Korteweg de Vries equation, by Zakharov and Faddeev in 1971. See [21]. The formula we use appears in a 1974 paper of Zakharov and Manakov; see [22]. This formula is useful both for the design of pulse sequences as well as for their analysis, as it allows for an a posteriori determination of the energy efficiency of a preexisting pulse sequence. 
Energy is just one among several important characteristics of a selective RF-pulse. Duration, maximum amplitude and rephasing time are also important parameters. While the minimum energy pulse may not be the best pulse for every application, it represents a good starting point when attempting to find that "best pulse." Beyond this, the inverse scattering method provides very direct control over the magnetization profile as well as these other parameters, allowing for the systematic design and analysis of RF-pulse sequences.

Underlying the derivation of the SLR algorithm is the so called hard pulse approximation. One designs a pulse envelope that is a finite sum of $\delta$-pulses which produces an approximation to the desired magnetization profile. As $\delta$-pulses are nonphysical, requiring infinite energy, an SLR pulse envelope is implemented by replacing the train of $\delta$-pulses by a train of boxcar pulses. The IST algorithm instead constructs a smooth pulse envelope which again produces an approximation to the desired magnetization profile. While such pulses are again implemented as a finite train of boxcar pulses, the approximations used in the IST are far less singular than those used in the SLR. Indeed the IST provides more direct and greater control over the final pulse envelope than the SLR. When I undertook this work the central mystery for me was why Shinnar-Le Roux has remained the industry standard and the inverse scattering transform is largely ignored. In addition to presenting the results, which seem interesting in and of themselves, my second purpose in writing this paper is to present the IST approach in a more explicit and palatable form. This accounts, in part, for the somewhat expository nature of our presentation.

In the first and second sections we recall the connection between the Bloch equation without relaxation and their spin domain formulation. In inverse scattering the spin domain Bloch equation is called the Zakharov-Shabbat $2 \times 2$-system or ZS-system. We next review the scattering theory for the ZS-system and relate the scattering data to the magnetization profile. These sections closely follow [14], [13] and [1]. In the third section we outline the inverse scattering transform via the Gel'fand-Levitan-Marchenko equation, or more briefly the Marchenko equation, following the treatment in [1]. In the fourth section we state the formula relating the energy in the RF-envelope to the magnetization profile and the location of the bound states. In the fifth section we discuss the problem of implementing the IST, and give a practical algorithm for solving the Marchenko equation. In the sixth section we address the problem of rephasing and the physical significance of the time parameter. In the seventh section we analyze the approximations used in both the SLR and IST approaches. The eighth section contains a variety of examples. Proofs of several mathematical results are contained in an Appendix.

\section{Acknowledgments}

I would like to thank Felix Wehrli for inviting me to visit the LSNI and giving me the opportunity to explore this fascinating subject. What I learned about magnetic resonance in conversations with Felix, Peter Joseph, Steve Pickup, Hee Kwon Song and Alex Wright has been indispensable in this work. Peter Joseph read through the entire manuscript and provided very helpful suggestions. I would also like to thank Carlos Tomei for all he has taught me over the years about inverse scattering. John D'Angelo provided some useful comments on the zeros of analytic functions. 


\section{The Spin Domain Bloch Equation and the Problem of RF-Pulse Synthesis}

The Bloch equation without relaxation is usually written in the form

$$
\frac{d \boldsymbol{M}}{d t}=\gamma \boldsymbol{M} \times \boldsymbol{B} .
$$

Here $\boldsymbol{M}$ is the magnetization, $\boldsymbol{B}$ is the applied magnetic field, $t$ is time and $\gamma$ is the gyromagnetic ratio. Since a vector evolves with constant length under this equation, the fundamental solution $U_{0}(t)$ can be regarded as $\boldsymbol{S} \boldsymbol{O}(3)$-valued function of time. Here we normalize so that $U_{0}(0)$ is $\mathrm{Id}_{3}$, the $3 \times 3$ identity matrix. If $\boldsymbol{M}(0)=\boldsymbol{M}_{0}$ then $\boldsymbol{M}(t)=U_{0}(t) \boldsymbol{M}_{0}$. The solution to the Bloch equation is linear in the initial data. Throughout this paper we assume that solutions of the Bloch equation are normalized to have length equal to one.

The Bloch equation is usually analyzed in a "rotating reference" frame. Ordinarily the rotating reference frame is related to the "laboratory frame" by a time dependent orthogonal transformation of the form

$$
\boldsymbol{F}(t)=\left[\begin{array}{ccc}
\cos \theta(t) & -\sin \theta(t) & 0 \\
\sin \theta(t) & \cos \theta(t) & 0 \\
0 & 0 & 1
\end{array}\right],
$$

so that

$$
\boldsymbol{M}(t)=\boldsymbol{F}(t) \boldsymbol{m}(t) .
$$

We use $\boldsymbol{m}$ to denote the magnetization in the rotating reference frame. Larmor's theorem implies that if $\boldsymbol{M}$ satisfies (1) then $\boldsymbol{m}$ satisfies

$$
\frac{d \boldsymbol{m}}{d t}=\gamma \boldsymbol{m} \times \boldsymbol{B}_{\mathrm{eff}}
$$

where

$$
\boldsymbol{B}_{\text {eff }}(t)=\boldsymbol{F}^{-1}(t) \boldsymbol{B}(t)+\frac{1}{\gamma} \boldsymbol{\Omega}(t) \quad \text { with } \boldsymbol{\Omega}(t)=\left[0,0, \theta^{\prime}(t)\right]^{\dagger} .
$$

Notational remark: Most of the vectors used in this paper are to be thought of a column vectors. The notation $[a, b, c]^{\dagger}$ refers to the transpose of the row vector which is therefore a column vector, i.e.

$$
[a, b, c]^{\dagger}=\left[\begin{array}{l}
a \\
b \\
c
\end{array}\right] .
$$

In most applications of this method the function $\theta(t)$ is selected to render the $z$ component of $\boldsymbol{B}_{\text {eff }}$ independent of time,

$$
\boldsymbol{B}_{\mathrm{eff}}(f ; t)=\left(\omega_{1}(t), \omega_{2}(t), \gamma^{-1} f\right) .
$$


The constant value $f$ is called the offset frequency or resonance offset. If we set $\omega(t)=$ $\omega_{1}(t)+i \omega_{2}(t)$ then, in the laboratory frame, the RF-envelope takes the form:

$$
\boldsymbol{p}(t)=\left[\operatorname{Re} \omega(t) e^{i \theta(t)}, \operatorname{Im} \omega(t) e^{i \theta(t)}, 0\right]^{\dagger} .
$$

The energy in the RF-envelope is given by

$$
W_{p}=\int_{-\infty}^{\infty}|\boldsymbol{p}(t)|^{2} d t=\int_{-\infty}^{\infty}|\boldsymbol{\omega}(t)|^{2} d t
$$

The magnetization profile is a unit vector valued function defined for $f \in \mathbb{R}$,

$$
\boldsymbol{m}^{\infty}(f)=\left[\begin{array}{l}
m_{1}^{\infty}(f) \\
m_{2}^{\infty}(f) \\
m_{3}^{\infty}(f)
\end{array}\right] .
$$

In essentially all MR applications $\boldsymbol{m}^{\infty}(f)=[0,0,1]^{\dagger}$ for $f$ outside of a bounded interval. The problem of RF-pulse synthesis is to find a time dependent complex pulse envelope $\boldsymbol{\omega}(t)$ so that, if $\boldsymbol{B}_{\text {eff }}(f)$ is given by (5), then the solution of

$$
\frac{d \boldsymbol{m}}{d t}(f ; t)=\gamma \boldsymbol{m}(f ; t) \times \boldsymbol{B}_{\mathrm{eff}}(f ; t),
$$

with

$$
\lim _{t \rightarrow-\infty} \boldsymbol{m}(f ; t)=[0,0,1]^{\dagger}
$$

satisfies

$$
\lim _{t \rightarrow \infty}\left[e^{-i f t}\left(m_{1}+i m_{2}\right)(f ; t), m_{3}(f ; t)\right]=\left[\left(m_{1}^{\infty}+i m_{2}^{\infty}\right)(f), m_{3}^{\infty}(f)\right] .
$$

We have used the standard complex notation for the transverse components of the magnetization. If $\omega$ is supported in the interval $\left[t_{0}, t_{1}\right]$, then these asymptotic conditions are replaced by

$$
\begin{aligned}
\boldsymbol{m}\left(f ; t_{0}\right) & =[0,0,1]^{\dagger} \\
{\left[e^{-i f t_{1}}\left(m_{1}+i m_{2}\right)\left(f ; t_{1}\right), m_{3}\left(f ; t_{1}\right)\right] } & =\left[\left(m_{1}^{\infty}+i m_{2}^{\infty}\right)(f), m_{3}^{\infty}(f)\right] .
\end{aligned}
$$

The mapping from $\omega$ to $\boldsymbol{m}^{\infty}$ is highly nonlinear; the problem of pulse synthesis is that of inverting this mapping.

To solve the problem of RF-pulse synthesis it is convenient to introduce the spin domain formulation of the Bloch equation. Instead of a unit vector $\boldsymbol{m}$ in $\mathbb{R}^{3}$ we solve for a unit vector $\boldsymbol{\psi}$ in $\mathbb{C}^{2}$. This vector satisfies the $2 \times 2$ matrix equation:

$$
\frac{d \psi}{d t}=-\frac{i}{2} \boldsymbol{Q} \cdot \boldsymbol{\sigma} \psi
$$

Here $\boldsymbol{Q}=-\left[\gamma \omega_{1}(t), \gamma \omega_{2}(t), f\right]$ and $\boldsymbol{\sigma}$ are the Pauli spin matrices:

$$
\sigma_{1}=\left[\begin{array}{ll}
0 & 1 \\
1 & 0
\end{array}\right], \quad \sigma_{2}=\left[\begin{array}{cc}
0 & -i \\
i & 0
\end{array}\right], \quad \sigma_{3}=\left[\begin{array}{cc}
1 & 0 \\
0 & -1
\end{array}\right] .
$$


Assembling the pieces we see that $\psi$ satisfies $^{1}$

$$
\frac{d \psi}{d t}(\xi ; t)=\left[\begin{array}{cc}
-i \xi & q(t) \\
-q^{*}(t) & i \xi
\end{array}\right] \psi(\xi ; t)
$$

with

$$
\xi=\frac{f}{2}, \quad q(t)=\frac{-i \gamma}{2}\left(\omega_{1}(t)-i \omega_{2}(t)\right) .
$$

A simple recipe takes a solution of (12) and produces a solution of (7). If $\psi(\xi ; t)=$ $\left[\psi_{1}(\xi ; t), \psi_{2}(\xi ; t)\right]^{\dagger}$ satisfies (12) then the 3 -vector

$$
\boldsymbol{m}(f ; t)=\left[2 \operatorname{Re}\left(\psi_{1}^{*} \psi_{2}\right), 2 \operatorname{Im}\left(\psi_{1}^{*} \psi_{2}\right),\left|\psi_{1}\right|^{2}-\left|\psi_{2}\right|^{2}\right]^{\dagger}\left(\frac{f}{2} ; t\right)
$$

satisfies (7). If in addition

$$
\lim _{t \rightarrow-\infty} \psi(\xi ; t)=\left[\begin{array}{c}
e^{-i \xi t} \\
0
\end{array}\right]
$$

then $\boldsymbol{m}$ satisfies (8). Thus the RF-pulse synthesis problem is easily translated into a inverse scattering problem for equation (12). This is described in the next section. Following the standard practice in inverse scattering we refer to equation (12) as the $Z S$-system and $q$ as the potential.

\section{Scattering Theory for the ZS-System}

Scattering theory for an equation like (12) relates the behavior of $\lim _{t \rightarrow-\infty} \psi(\xi ; t)$ to that of $\lim _{t \rightarrow \infty} \psi(\xi ; t)$. If $q$ has bounded support, then the functions

$$
\left[\begin{array}{c}
e^{-i \xi t} \\
0
\end{array}\right], \quad\left[\begin{array}{c}
0 \\
e^{i \xi t}
\end{array}\right]
$$

are a basis of solutions for (12) outside the support of $q$. If the $L^{1}$-norm of $q$ is finite, then it is shown in [1] that (12) has solutions which are asymptotic to these solutions as $t \rightarrow \pm \infty$.

Theorem 1. If $\|q\|_{L^{1}}$ is finite, then, for every real $\xi$, there are unique solutions

$$
\boldsymbol{\psi}_{1+}(\xi), \boldsymbol{\psi}_{2+}(\xi) \text { and } \boldsymbol{\psi}_{1-}(\xi), \boldsymbol{\psi}_{2-}(\xi)
$$

to equation (12) which satisfy

$$
\begin{gathered}
\lim _{t \rightarrow-\infty} e^{i \xi t} \boldsymbol{\psi}_{1-}(\xi ; t)=\left[\begin{array}{l}
1 \\
0
\end{array}\right], \quad \lim _{t \rightarrow-\infty} e^{-i \xi t} \boldsymbol{\psi}_{2-}(\xi ; t)=\left[\begin{array}{c}
0 \\
-1
\end{array}\right] \\
\lim _{t \rightarrow \infty} e^{i \xi t} \boldsymbol{\psi}_{1+}(\xi ; t)=\left[\begin{array}{l}
1 \\
0
\end{array}\right], \quad \lim _{t \rightarrow \infty} e^{-i \xi t} \boldsymbol{\psi}_{2+}(\xi ; t)=\left[\begin{array}{l}
0 \\
1
\end{array}\right]
\end{gathered}
$$

The solutions $\boldsymbol{\psi}_{1-}(\xi), \boldsymbol{\psi}_{2+}(\xi)$ extend as analytic functions of $\xi$ to the upper half plane, $\operatorname{Im} \xi>0$ and $\psi_{2-}(\xi), \psi_{1+}(\xi)$ extend as analytic functions of $\xi$ to the lower half plane, $\operatorname{Im} \xi<0$.

\footnotetext{
${ }^{1}$ We follow the standard practice in the MR literature of using $z^{*}$ to denote the complex conjugate of the complex number $z$.
} 
2 Scattering Theory for the ZS-System

The proof of this theorem can be found in [1]. We briefly outline the construction of these solutions as we will need these formulæ later on.

Outline of proof. The idea is to reexpress the boundary value problems for the differential equation as Volterra integral equations. Let

$$
\psi_{1-}=\left[\begin{array}{l}
\psi_{11-} \\
\psi_{21-}
\end{array}\right], \quad \psi_{2-}=\left[\begin{array}{l}
\psi_{12-} \\
\psi_{22-}
\end{array}\right] .
$$

These solutions satisfy:

$$
\begin{aligned}
\psi_{11-}(\xi ; t) e^{i \xi t} & =1+\int_{-\infty}^{t} M_{1}(\xi ; t, s) \psi_{11-}(\xi ; s) e^{i \xi s} d s \\
\psi_{21-}(\xi ; t) e^{i \xi t} & =-\int_{-\infty}^{t} e^{2 i \xi(t-s)} q^{*}(s) \psi_{11-}(\xi ; s) e^{i \xi s} d s, \\
\psi_{22-}(\xi ; t) e^{-i \xi t} & =-1+\int_{-\infty}^{t} M_{2}(\xi ; t, s) \psi_{22-}(\xi ; s) e^{-i \xi s} d s, \\
\psi_{12-}(\xi ; t) e^{-i \xi t} & =-\int_{-\infty}^{t} e^{-2 i \xi(t-s)} q(s) \psi_{22-}(\xi ; s) e^{-i \xi s} d s
\end{aligned}
$$

where

$$
\begin{aligned}
& M_{1}(\xi ; t, s)=-q^{*}(s) \int_{s}^{t} e^{2 i \xi(x-s)} q(x) d x \\
& M_{2}(\xi ; t, s)=-q(s) \int_{s}^{t} e^{2 i \xi(s-x)} q^{*}(x) d x .
\end{aligned}
$$

The solutions normalized at $+\infty$ satisfy similar equations. If we set

$$
Q_{1}(t)=\int_{-\infty}^{t}|q(s)| d s
$$

then it is shown in [1] that these equations can be solved for $\xi$ in the appropriate half plane by iteration and

$$
\left|\psi_{11-}(\xi ; t) e^{i \xi t}\right| \leq I_{0}\left(2 Q_{1}(t)\right), \quad\left|\psi_{22-}(\xi ; t) e^{-i \xi t}\right| \leq I_{0}\left(2 Q_{1}(t)\right) .
$$

Here $I_{0}$ is the classical $I_{0}$-Bessel function. The same argument applies mutatis mutandis to obtain $\psi_{1+}$ and $\psi_{2+}$. 
For real values of $\xi$, the solutions normalized at $-\infty$ can be expressed in terms of the solutions normalized at $+\infty$ by the linear relations

$$
\begin{array}{r}
\boldsymbol{\psi}_{1-}(\xi ; t)=a(\xi) \boldsymbol{\psi}_{1+}(\xi ; t)+b(\xi) \boldsymbol{\psi}_{2+}(\xi ; t) \\
\boldsymbol{\psi}_{2-}(\xi ; t)=b^{*}(\xi) \boldsymbol{\psi}_{1+}(\xi ; t)-a^{*}(\xi) \boldsymbol{\psi}_{2+}(\xi ; t)
\end{array}
$$

The functions $a, b$ are called the scattering coefficients for the potential $q$. The $2 \times 2$ matrices $\left[\boldsymbol{\psi}_{1-} \boldsymbol{\psi}_{2-}\right],\left[\boldsymbol{\psi}_{1+} \boldsymbol{\psi}_{2+}\right]$ satisfy

$$
\left[\boldsymbol{\psi}_{1-} \boldsymbol{\psi}_{2-}\right]=\left[\boldsymbol{\psi}_{1+} \boldsymbol{\psi}_{2+}\right]\left[\begin{array}{cc}
a(\xi) & b^{*}(\xi) \\
b(\xi) & -a^{*}(\xi)
\end{array}\right] .
$$

The scattering matrix for the potential $q$ is defined to be

$$
s(\xi)=\left[\begin{array}{cc}
a(\xi) & b^{*}(\xi) \\
b(\xi) & -a^{*}(\xi)
\end{array}\right] .
$$

Recall that the Wronskian between two $\mathbb{C}^{2}$-valued functions of $t$ is defined by

$$
W(\boldsymbol{u}, \boldsymbol{v})(t) \stackrel{d}{=} u_{1}(t) v_{2}(t)-u_{2}(t) v_{1}(t)
$$

If $\boldsymbol{u}(t)$ and $\boldsymbol{v}(t)$ are solutions of (12), for the same value of $\xi$, then $W(\boldsymbol{u}(t), \boldsymbol{v}(t))$ is independent of $t$. It is not difficult to show that

$$
\begin{aligned}
& a(\xi)=\left[\psi_{11-}(\xi ; t) \psi_{22+}(\xi ; t)-\psi_{21-}(\xi ; t) \psi_{12+}(\xi ; t)\right]=W\left(\boldsymbol{\psi}_{1-}, \boldsymbol{\psi}_{2+}\right)(t), \\
& b(\xi)=-\left[\psi_{11-}(\xi ; t) \psi_{21+}(\xi ; t)-\psi_{21-}(\xi ; t) \psi_{11+}(\xi ; t)\right]=-W\left(\boldsymbol{\psi}_{1-}, \boldsymbol{\psi}_{1+}\right)(t)
\end{aligned}
$$

It follows from (22) that $a$ extends to the upper half plane as an analytic function. If $q$ has an integrable derivative, then, using (16) and (22), it can be shown that

$$
a(\xi)=1+\frac{1}{2 i \xi} \int_{-\infty}^{\infty}|q(s)|^{2} d s+O\left(\frac{1}{\xi^{2}}\right),
$$

as $|\xi|$ tends to infinity in $\operatorname{Im} \xi \geq 0$. As $W\left(\boldsymbol{\psi}_{1-}, \boldsymbol{\psi}_{2-}\right)=-1$ it follows that

$$
|a(\xi)|^{2}+|b(\xi)|^{2}=1,
$$

and therefore (23) implies that

$$
|b(\xi)|=O\left(\frac{1}{|\xi|}\right) \quad \text { as } \xi \rightarrow \pm \infty .
$$

These results are proved in [1].

Assuming that $t^{j} q(t)$ is integrable for all $j$ it is shown in [1] that $a$ has finitely many zeros in $\operatorname{Im} \xi \geq 0$. Let $\left\{\xi_{1}, \ldots, \xi_{N}\right\}$ be a list of the zeros of $a$. For each $j$ it follows from (22) that there is a nonzero complex number $C_{j}^{\prime}$ so that

$$
\boldsymbol{\psi}_{1-}\left(\xi_{j}\right)=C_{j}^{\prime} \psi_{2+}\left(\xi_{j}\right), \quad j=1, \ldots, N .
$$


Equation (12) can be rewritten in the form

$$
\left[\begin{array}{cc}
i \partial_{t} & -i q \\
-i q^{*} & -i \partial_{t}
\end{array}\right]\left[\begin{array}{l}
\psi_{1} \\
\psi_{2}
\end{array}\right]=\xi\left[\begin{array}{l}
\psi_{1} \\
\psi_{2}
\end{array}\right]
$$

From this formulation it is clear that $\xi$ should be regarded as a spectral parameter. If $\xi$ has positive imaginary part, then $\psi_{1-}(\xi ; t)$ decays exponentially as $t$ tends to $-\infty$ and $\psi_{2+}(\xi ; t)$ decays exponentially as $t$ tends to $+\infty$. If $a\left(\xi_{j}\right)=0$, then (26) implies that $\psi_{1-}\left(\xi_{j} ; t\right)$ decays exponentially at both $\pm \infty$ and therefore the function $\psi_{1-}\left(\xi_{j} ; t\right)$ belongs to $L^{2}\left(\mathbb{R} ; \mathbb{C}^{2}\right)$. Thus the operator on the left hand side of (27) has bound states for these values of the offset frequency.

We generally assume that the zeros of $a$ are simple and that their imaginary parts are positive. This is mostly to simplify the exposition, there is no difficulty, in principle, if $a$ has real zeros or higher order zeros. Using the argument principle and (23) it follows that

$$
N=\frac{1}{2 \pi i} \int_{-\infty}^{\infty} \frac{a^{\prime}(\xi) d \xi}{a(\xi)}
$$

Definition 1. The pair of functions $(a(\xi), b(\xi))$, for $\xi \in \mathbb{R}$, and the collection of pairs $\left\{\left(\xi_{j}, C_{j}\right): j=1, \ldots, N\right\}$ define the scattering data for equation (12).

The scattering data are not quite independent. If $a$ has zeros at $\left\{\xi_{j}\right\}$ then

$$
\tilde{a}(\xi)=\prod_{j=1}^{N}\left(\frac{\xi-\xi_{j}^{*}}{\xi-\xi_{j}}\right) a(\xi)
$$

is analytic and nonvanishing in the upper half plane. Moreover $|a(\xi)|=|\tilde{a}(\xi)|$ on the real axis. The function $\log \tilde{a}$ is also analytic in the upper half plane and the asymptotic formula (23) implies that $|\log \tilde{a}(\xi)|$ is $O\left(|\xi|^{-1}\right)$ as $|\xi|$ tends to infinity. The Cauchy integral formula therefore applies to give a representation of $\log \tilde{a}$ in $\operatorname{Im} \xi>0$ :

$$
\log \tilde{a}(\xi)=\frac{1}{2 \pi i} \int_{-\infty}^{\infty} \frac{\log \left(|a(\zeta)|^{2}\right) d \zeta}{\zeta-\xi} .
$$

Exponentiating and putting the zeros of $a$ back in gives

$$
a(\xi)=\prod_{j=1}^{N}\left(\frac{\xi-\xi_{j}}{\xi-\xi_{j}^{*}}\right) \exp \left[\frac{1}{2 \pi i} \int_{-\infty}^{\infty} \frac{\log \left(|a(\zeta)|^{2}\right) d \zeta}{\zeta-\xi}\right],
$$

see [8]. The reflection coefficient is defined by

$$
r(\xi)=\frac{b(\xi)}{a(\xi)} .
$$

A priori the reflection coefficient is only defined on the real axis. Using (24) we rewrite (29) in terms of $r$ :

$$
a(\xi)=\prod_{j=1}^{n}\left(\frac{\xi-\xi_{j}}{\xi-\xi_{j}^{*}}\right) \exp \left[\frac{i}{2 \pi} \int_{-\infty}^{\infty} \frac{\log \left(1+|r(\zeta)|^{2}\right) d \zeta}{\zeta-\xi}\right] .
$$


Both (29) and (31) have well defined limits as $\xi$ approaches the real axis.

If $a$ has simple zeros at the points $\left\{\xi_{1}, \ldots, \xi_{N}\right\}$ then we define the norming constants by setting

$$
C_{j}=\frac{C_{j}^{\prime}}{a^{\prime}\left(\xi_{j}\right)},
$$

where the $\left\{C_{j}^{\prime}\right\}$ are defined in (26). The definition needs to be modified if $a$ has nonsimple zeros. The reason for replacing $\left\{C_{j}^{\prime}\right\}$ with $\left\{C_{j}\right\}$ will become more apparent in section 3.

Definition 2. The function $r(\xi)$, for $\xi \in \mathbb{R}$ and the collection of pairs $\left\{\left(\xi_{j}, C_{j}\right): j=\right.$ $1, \ldots, N\}$ define the reduced scattering data for equation (12).

Implicitly the reduced scattering data is a function of the potential $q$. In inverse scattering theory, the data $\left\{r ;\left(\xi_{1}, C_{1}\right), \ldots,\left(\xi_{N}, C_{N}\right)\right\}$ are specified, and we seek a potential $q$ which has this reduced scattering data. The map from this data to $q$ is often called the Inverse Scattering Transform or IST.

We close this section by rephrasing the RF-pulse synthesis problem as an inverse scattering problem. Recall that the data for the pulse synthesis problem is the magnetization profile $\boldsymbol{m}^{\infty}$, which we now think of as a function of $\xi=f / 2$. Using (13), the solution $\boldsymbol{\psi}_{1-}$ to the ZS-system defines a solution $\boldsymbol{m}_{1-}$ to (7), satisfying (8). It follows from (15) and (19) that

$$
\psi_{1-}(\xi ; t) \sim\left[\begin{array}{c}
a(\xi) e^{-i \xi t} \\
b(\xi) e^{i \xi t}
\end{array}\right] \quad \text { as } t \rightarrow+\infty
$$

Therefore

$$
\boldsymbol{m}_{1-}(\xi ; t) \sim\left[\begin{array}{c}
2 b(\xi) a^{*}(\xi) e^{2 i \xi t} \\
|a(\xi)|^{2}-|b(\xi)|^{2}
\end{array}\right]
$$

where, as before, we use the complex notation for the transverse components of $\boldsymbol{m}_{1-}$. If $\boldsymbol{m}_{1-}$ also satisfies (9) then it follows from (34) and (24) that

$$
\begin{aligned}
\frac{b(\xi)}{a(\xi)} & =\lim _{t \rightarrow \infty} \frac{\left(m_{11-}+i m_{21-}\right)(\xi ; t) e^{-2 i \xi t}}{1+m_{31-}(\xi ; t)} \\
& =\frac{\left(m_{1}^{\infty}+i m_{2}^{\infty}\right)(\xi)}{1+m_{3}^{\infty}(\xi)} .
\end{aligned}
$$

In light of this formula, we sometimes refer to $r$ as the stereographic magnetization profile. If $q$ has support in the ray $\left(-\infty, t_{1}\right]$ then

$$
r(\xi)=\frac{\left(m_{11-}+i m_{21-}\right)(\xi ; t) e^{-2 i \xi t}}{1+m_{31-}(\xi ; t)}
$$

is independent of $t$ for $t>t_{1}$.

As $\boldsymbol{m}^{\infty}$ is a unit vector valued function, we see that the reflection coefficient $r(\xi)$ uniquely determines $\boldsymbol{m}^{\infty}(\xi)$ and vice-versa. Thus the RF-pulse synthesis problem can be rephrased as the following inverse scattering problem: 
Find a potential $q$ for the ZS-system so that the reflection coefficient $r$ satisfies (35) for all real $\xi$.

Note that the pulse synthesis problem makes no reference to the data connected to the bound states, i.e. $\left\{\left(\xi_{j}, C_{j}\right)\right\}$. Indeed these are free parameters in the RF pulse synthesis problem, making the problem highly under-determined. Perhaps the main result of this paper is that, for a given magnetization profile, the RF-envelope requiring the minimum energy is the one for which the ZS-system has no bound states.

Remark 1. The material in this section is largely adapted from [1], [14] and [13].

\section{Inverse Scattering for the ZS-System}

There are several different approaches to solving the inverse scattering problem stated at the end of the previous section, see [1], [2], [4] or [8]. In this section we describe the solution of this problem via the Marchenko equations. We again follow the presentation in [1]. We generally suppose that the $\left\{\xi_{j}\right\}$ are distinct complex numbers with positive imaginary parts. This simplifies the exposition, it is not necessary for the applicability of the inverse scattering method. To begin with, we assume that the reflection coefficient is smooth and rapidly vanishing at $\pm \infty$. Using limiting arguments, the latter restriction is easily relaxed.

The Marchenko equations are systems of integral equations of Fredholm type. Given the reduced scattering data $\left\{r(\xi), \xi \in \mathbb{R} ;\left(\xi_{1}, C_{1}\right), \ldots,\left(\xi_{N}, C_{N}\right)\right\}$, with $\xi_{i} \neq$ $\xi_{j}$, for $i \neq j$, define the function

$$
f(t)=\frac{1}{2 \pi} \int_{-\infty}^{\infty} r(\xi) e^{i \xi t} d \xi-i \sum_{j=1}^{N} C_{j} e^{i \xi_{j} t}
$$

This is the inverse Fourier transform of $r$ with a correction added to account for the bound states. The finite sum in (37) is exponentially decreasing as $t$ tends to $+\infty$. Define the $2 \times 2$ matrix valued function:

$$
\mathscr{F}(t)=\left[\begin{array}{cc}
0 & -f^{*}(t) \\
f(t) & 0
\end{array}\right]
$$

For each number $t \in \mathbb{R}$, we seek a $2 \times 2$-matrix valued function, $\mathscr{K}_{t}(s)$, defined for $s \in[t, \infty)$, which solves the integral equation:

$$
\mathscr{K}_{t}(s)+\int_{t}^{\infty} \mathscr{K}_{t}(\sigma) \mathscr{F}(s+\sigma) d \sigma=-\mathscr{F}(t+s) \text { for } s \in[t, \infty) \text {. }
$$

Under our assumptions on $r$ it is not difficult to show that, for every $t \in \mathbb{R}$, (38) is a Fredholm integral equation on $L^{2}\left([t, \infty) ; \mathfrak{g} l_{2}\right)$. As $\mathscr{F}(t+\cdot)$ belongs to $L^{2}\left([t, \infty) ; \mathfrak{g} l_{2}\right)$, 
the solvability of (38) follows if it can be shown that any solution to the homogeneous adjoint equation,

$$
\mathscr{L}(s)+\int_{t}^{\infty} \mathscr{L}(\sigma) \mathscr{F}^{*}(s+\sigma) d \sigma=0,
$$

is identically zero. This proof of this statement can be found in [1]. The uniqueness of the solution to (38) follows from the fact that homogeneous equation corresponding to (38) also has only the trivial solution.

Remark 2. For the theory of Fredholm integral equations see [20]. For the moment we note that the known proofs for the existence of a solution to a Fredholm equation do not generally provide a practicable algorithm to find the solution.

The connection between the inverse scattering problem and the Marchenko equation is summarized in the following theorem:

Theorem 2. Given a smooth, rapidly decaying reflection coefficient $r$, and a finite set of pairs $\left\{\left(\xi_{j}, C_{j}\right): j=1, \ldots, N\right\}$, with the $\left\{\xi_{j}\right\}$ distinct, $\operatorname{Im} \xi_{j}>0$ and $C_{j} \neq 0$ for $j=1, \ldots, N$, the equation (38) has a unique solution for every $t \in \mathbb{R}$. If

$$
q(t)=-2 \mathcal{K}_{12 t}(t),
$$

then the ZS-system with this potential has reflection coefficient $r$. It has exactly $N$ bound states for frequencies $\left\{\xi_{1}, \ldots, \xi_{N}\right\}$ and the relations (26) hold at these points.

This theorem is proved in [8] and, in a slightly different formulation in [2].

Remark 3. From the theorem we see that it is only necessary to solve for $\mathscr{K}_{12 t}(s)$. This function satisfies a scalar integral equation:

$$
\mathscr{K}_{12 t}(s)+\int_{t}^{\infty}\left[\int_{t}^{\infty} \mathscr{K}_{12 t}(x) f(x+y) d x\right] f^{*}(y+s) d y=f^{*}(t+s) .
$$

This equation can be rewritten in the form

$$
\left[\left(\mathrm{Id}+F_{t}^{*} F_{t}\right) \mathscr{K}_{12 t}\right](s)=f^{*}(t+s),
$$

where the operator $F_{t}$ is defined by

$$
F_{t} h(s)=\int_{t}^{\infty} f(s+y) h(y) d y
$$

As the operator $\left(\mathrm{Id}+F_{t}^{*} F_{t}\right)$ is self adjoint and positive definite, it is an elementary fact that (40) has a unique solution. Since $F_{t}$ is a compact operator, it is straightforward to numerically approximate this equation. We defer a discussion of the practicalities of solving (38) to Section 5. For the moment suffice it to say that this equation can be solved by a simple iteration, for values of $t$, such that

$$
\int_{2 t}^{\infty}|f(x)| d x<1
$$


A little more care is required for smaller values of $t$. There is also a "left" Marchenko equation, given in Section 5, where the integrals are over half lines of the form $(-\infty, t]$. In this formulation, the analogous finite sum of exponentials is decreasing as $t$ tends to $-\infty$. Using both the left and right Marchenko equations, it is fairly straightforward to obtain an efficient, stable algorithm for solving (40). The algorithm works equally well whether or not there are bound states.

Remark 4. As noted above, there is a similar result if $a$ has higher order zeros or zeros on the real line. In this case the definition of $f$ needs to be modified; see [1].

Remark 5. In the Fourier transform method of pulse design, the excitation profile (as a function of the offset frequency $f$ ), and the RF-envelope are viewed as a Fourier transform pair. This theory is asymptotically correct as the flip angle goes to zero. The inverse scattering method suggests an improved first order theory: While the potential $q$ depends nonlinearly on $f$, the leading order, or linear term, is

$$
q^{1}(t)=-2 f^{*}(2 t)=-2[\check{r}(2 t)]^{*}
$$

where $\check{r}$ is the inverse Fourier transform of $r$. The factors of 2 arise because $r$ is a function of $\xi=\frac{f}{2}$.

The regularity and decay of $q$ are related to the regularity and decay of $r$ exactly as if they were a Fourier transform pair. The following result is a special case of a theorem proved in [2]:

Theorem 3 (Beals-Coifman). Suppose that $r$ is a reflection coefficient and $q$ is the corresponding potential for the ZS-system. Let $k$ be a positive integer.

1. If

$$
\int_{-\infty}^{\infty}\left[|\xi|^{2 k}+1\right]|r(\xi)|^{2} d \xi<\infty
$$

then the distributional derivatives $\partial_{t}^{j} q$, for $0 \leq j \leq k$, belong to $L^{2}(\mathbb{R})+$ $L^{\infty}(\mathbb{R})$.

2. If the distribution derivatives $\partial_{\xi}^{j} r \in L^{2}(\mathbb{R})$ for $0<j \leq k+1$, then $t^{k+1} q \in$ $L^{2}(\mathbb{R})+L^{\infty}(\mathbb{R})$.

Remark 6. There are no smallness hypotheses in this theorem. Note also that this theorem does not mention the bound states. While the bound states exercise a strong effect on the detailed structure of $q$, their presence or absence has no effect on the gross smoothness and decay properties of the potential. These features of $q$ are governed entirely by the reflection coefficient.

Remark 7. It has been noted many times in both the inverse scattering and MR literatures that, as $q$ tends to zero, $r^{*}(\xi)$ tends to $\hat{q}(-2 \xi)$. The results of Beals and Coifman shows that the connection between the inverse scattering transform and the Fourier transform is much deeper than that previously described. There is also an analogue of the Paley-Wiener theorem (see the Appendix): If the reflection coefficient has bounded 
support, then the potential does not. This is easier to see in the logically equivalent contrapositive form: If the potential has bounded support, then the reflection coefficient does not. When the potential has bounded support the scattering coefficients $(a, b)$ extend, as analytic functions, to the entire complex plane. This means that the set where $b$ (and therefore $r$ ) vanishes is discrete. Hence the reflection coefficient, restricted to the real axis, cannot have bounded support.

Remark 8. Beals and Coifman show that the IST can be extended to square integrable reflection coefficients such that $\partial_{\xi} r$ also belongs to $L^{2}(\mathbb{R})$. By using smooth, compactly support approximating sequences, the estimates in the theorem can be extended to data with finitely many derivatives and finite rates of decay.

The results of this section show that, at least in principle, the IST provides many solutions to the RF-pulse synthesis problem. A practical magnetization profile has bounded support and may be approximated by a smooth function. While the choice of approximation is quite important, we do not consider it systematically in this paper. This leaves only the question of choosing the bound states to obtain an "optimal" RF-pulse envelope, that will produce the desired magnetization profile. The notion of optimality depends on the intended application. It usually entails a balance among the energy, duration, oscillation and need for rephasing of the RF-envelope. In the remainder of the paper we focus on the construction of the minimum energy RF-envelope.

\section{The Energy of the RF-Envelope}

We now state a formula for the energy of the pulse envelope in terms of the reduced scattering data. The underlying results are due to Zakharov, Faddeev and Manakov.

Theorem 4. Suppose that $q$ is a sufficiently rapidly decaying potential for the ZSsystem with reflection coefficient $r$ and discrete data $\left\{\left(\xi_{j}, C_{j}\right), j=1, \ldots, N\right\}$, then

$$
\int_{-\infty}^{\infty}|q(t)|^{2} d t=\frac{1}{\pi} \int_{-\infty}^{\infty} \log \left(1+|r(\xi)|^{2}\right) d \xi+4 \sum_{j=1}^{N} \operatorname{Im} \xi_{j} .
$$

The proof of this result can be found in [1] or [8]. Note that the norming constants play no role in this formula. Formula (42) is just one from an infinite sequence of formulæ relating functionals of the potential to functionals of the reduced scattering data.

Combining (6), (42) with (35) we obtain the following simple corollary.

Corollary 1. If $\boldsymbol{m}^{\infty}$ is a sufficiently smooth magnetization profile such that $m_{1}^{\infty}+i m_{2}^{\infty}$ vanishes outside a finite interval, then the total energy of any RF-envelope, $\omega$, which produces this magnetization profile satisfies the estimate

$$
\int_{-\infty}^{\infty}|\omega(t)|^{2} d t \geq \frac{2}{\pi \gamma^{2}} \int_{-\infty}^{\infty} \log \left(1+\left|\frac{m_{1}^{\infty}(\xi)+i m_{2}^{\infty}(\xi)}{1+m_{3}^{\infty}(\xi)}\right|^{2}\right) d \xi .
$$

Equality holds in this estimate if and only if the ZS-system with the corresponding potential has no bound states. 
From the corollary it is evident that the lowest energy RF-envelope is obtained by solving the Marchenko equation with

$$
f(t)=\frac{1}{2 \pi} \int_{-\infty}^{\infty} r(\xi) e^{i \xi t} d \xi .
$$

For $x>0$ we have the inequality $\log (1+x)<x$, and therefore, for the (nonzero) minimum energy pulse,

$$
\begin{aligned}
\int_{-\infty}^{\infty}|q(t)|^{2} d t & =\frac{1}{\pi} \int_{-\infty}^{\infty} \log \left(1+|r(\xi)|^{2}\right) d \xi \\
& <\frac{1}{\pi} \int_{-\infty}^{\infty}|r(\xi)|^{2} d \xi
\end{aligned}
$$

Using Parseval's formula, this gives the estimate

$$
\int_{-\infty}^{\infty}|q(t)|^{2} d t<\int_{-\infty}^{\infty}\left|2 f^{*}(2 t)\right|^{2} d t
$$

Hence the minimum energy pulse always has less energy than the first order approximate solution. The $\log (1+x)$ grows much slower than $x$ and therefore, for a "large" $r$, (i.e., high flip angle) the energy in the minimum energy pulse is much smaller than that predicted by the linear theory.

Remark 9. There are several other immediate conclusions that can be drawn from this formula. Often times one seeks to specify a particular dependence for the phase of the transverse magnetization. Changing the phase of the transverse magnetization amounts to replacing $\left(m_{1}^{\infty}(\xi)+i m_{2}^{\infty}(\xi)\right)$ by $\left(m_{1}^{\infty}(\xi)+i m_{2}^{\infty}(\xi)\right) e^{i \phi(\xi)}$, where $\phi$ is a real valued function. From (43) it is apparent that the energy required for the minimum energy RFenvelope is independent of the phase of the transverse magnetization. Note however that the minimum energy pulse will in general require rephasing. This is addressed in Section 6.

Remark 10. Very important types of pulses in MR imaging are inversion or refocusing pulses. Such a pulse carries $[0,0,1]$ to $[0,0,-1]$. If this is the case at offset frequency $\xi_{0}$ then $r\left(\xi_{0}\right)=\infty$. It would therefore require infinite energy to exactly invert $[0,0,1]$ for offset frequencies belonging to an interval of positive length. The energy required to carry $[0,0,1]$ to a vector of the form $\left[m_{1}, m_{2},-1+e\right]$, for $0 \leq e<\epsilon$, and offset frequencies belonging to a band of width $B$, is at least

$$
\frac{2 B}{\pi \gamma^{2}} \log \left(1+\frac{4(2-\epsilon)}{\epsilon}\right) \text {. }
$$

We would also like to have a means for estimating the support of $q$ in terms of the reduced scattering data. In Section 5 we show that the effective support of $q$ is bounded 
by the effective support of the function $f$, defined in (37). Empirically, we find that the effective support of the minimum energy pulse is a proper subset of the effective support of $f$.

In the absence of bound states it is not hard to show that if $r(\xi)$ is the reflection coefficient determined by $q(t)$ then, for $\lambda>0, r(\lambda \xi)$ is the reflection coefficient determined by the potential $\lambda^{-1} q\left(\lambda^{-1} t\right)$. This agrees with the well known heuristic principle that the effective support of the RF-envelope is inversely proportional to the bandwidth of excitation. It also shows that the maximum amplitude and energy of the RF-envelope are proportional to the bandwidth. The $L^{2}$-oscillation of the RF-envelope, $\int\left|q_{t}\right|^{2} d t$, is proportional to the square of the bandwidth.

\section{Solving the Marchenko Equation}

In this section we discuss an algorithmic approach for obtaining the minimum energy RF-envelope. It entails solving the Marchenko equation, (38) and a related equation, (47), directly via iteration. As noted above, this requires some care as large data may lead to a divergent series. In [14] Rourke and Morris introduced a method for solving the Marchenko equation by adapting a method of Moses and Proesser, see [11]. The analysis in [14] differs in one small particular from that in [11], leading to solutions of the Marchenko equation with nontrivial bound states and therefore sub-optimal energy requirement.

The method of Moses and Proesser is to approximate the reflection coefficient by a rational function with all of it poles in the lower half plane. This ensures that the resultant potential is supported in $(-\infty, 0]$ and has no bound states. In [14] this condition on the placement of the poles is not respected. Indeed in examples 2 and 3 of [14] they use a rational approximation to $r$ with poles in both half planes; the solutions they find are supported in a proper subinterval of $(-\infty, 0)$. However the poles of $r$ in the upper half plane force the resultant potential for the ZS-system to have nontrivial bound states. These increase the energy and oscillation of the RF-envelope without affecting the magnetization profile. Regrettably the method of Moses and Proesser is not generally applicable to find the minimum energy RF-envelope. This is due to the well known consequence of the maximum principle in the theory of functions of a complex variable: A function on the real line, which tends to zero at infinity, is only well approximated by rational functions with poles in the lower half plane if it is the boundary value of an analytic function defined in the upper half plane. In light of a classical theorem of Fatou, this excludes any function supported in a bounded interval, see [15].

We are left with little choice but to solve the equation (40) directly. We now present an algorithm for doing this. The algorithm is described in the realm of continuum mathematics but can easily be approximately implemented on a computer. After stating the "left" Marchenko equation we give an estimate which shows that, for a given reflection coefficient, the Marchenko equations can be solved by a simple iteration for sufficiently large $|t|$. There are two ideas which underlie our algorithm:

1. Using the Marchenko equation we solve for the potential one $t$ at a time.

2. For each $t$, the operator $\left(\operatorname{Id}+F_{t}^{*} F_{t}\right)$ is Hermitian and positive definite. 
Using these ideas leads to a method which allows us to work inward from $\pm \infty$ to determine the potential for all values of $t$.

Theorem 2, and its extension to less regular data, imply that, given a sufficiently regular reflection coefficient $r$, there exists a potential $q$ so that the ZS-system, with this potential, has reflection coefficient $r$ and no bound states. In this section, the data $r$, and the corresponding potential $q$ are fixed. We assume that $r$ decays rapidly enough and is sufficiently regular for $q$ to be bounded and integrable with two continuous derivatives, see Theorem 3.

Equation (38) involves integration over positive half rays. Because of the divergences indicated above, it is useful to work both ends against the middle. For that purpose we give the left Marchenko equation which involves integration over negative half rays. For later applications we give the left Marchenko equation with finitely many bound states. Recall that $r=b / a$; given $r$, we can use (31) to determine $a$ and therefore $b$. Define

$$
\tilde{r}(\xi)=\frac{b^{*}(\xi)}{a(\xi)},
$$

and let $\left\{\xi_{1}, \ldots, \xi_{N}\right\}$ be the zeros of $a$ in the upper half plane. There are nonzero constants $\left\{\widetilde{C}_{j}\right\}$ so that the kernel function for the left Marchenko equation is defined in terms of

$$
g(t)=\frac{1}{2 \pi} \int_{-\infty}^{\infty} \tilde{r}(\xi) e^{-i \xi t} d t-i \sum_{j=1}^{N} \widetilde{C}_{j} e^{-i \xi_{j} t}
$$

The exponential correction terms are exponentially decaying as $t$ tends to $-\infty$. The kernel for the left Marchenko equation is

$$
\mathscr{G}(t)=\left[\begin{array}{cc}
0 & g(t) \\
-g^{*}(t) & 0
\end{array}\right] .
$$

For each fixed $t \in \mathbb{R}$, the equation is

$$
\mathscr{L}_{t}(s)+\int_{-\infty}^{t} \mathscr{L}_{t}(x) \mathscr{G}(x+s) d x=-\mathscr{G}(t+s) \quad \text { for } s \in(-\infty, t] .
$$

If $\mathscr{L}_{t}(s)$ solves this equation, then the potential defined by

$$
q(t)=2 \mathscr{L}_{12 t}(t)
$$

has reflection coefficient $r$, and bound states at $\left\{\xi_{j}\right\}$. Equation (47) can be derived from (38) by considering the scattering theory for the "time reversed" potential

$$
q_{-}(t)=-q(-t) .
$$

For example, it is not difficult to show that the reflection coefficient for $q_{-}$is

$$
r_{-}(\xi)=-\frac{b(-\xi)}{a^{*}(-\xi)} .
$$


In the remainder of our analysis we concentrate on the right Marchenko equation, (38), with the understanding that everything said applies, mutatis mutandis, to the left formulation as well. The method we outline below works whether or not there are bound states. A stable, efficient method for finding $q$ uses the right equation for positive values of $t$ and the left equation for negative values.

Let $\|\cdot\|$ denote an operator norm. This is a norm defined on linear transformations from a normed linear space $\left(X,\|\cdot\|_{X}\right)$ to a normed linear space $\left(Y,\|\cdot\|_{Y}\right)$ that satisfies:

$$
\|A \boldsymbol{x}\|_{Y} \leq\|A\|\|\boldsymbol{x}\|_{X}
$$

If $X=Y$ then this implies that

$$
\|A B\| \leq\|A\|\|B\|
$$

For example we can take

$$
\|A\|=\sup _{\boldsymbol{x} \neq 0} \frac{\|A \boldsymbol{x}\|_{Y}}{\|\boldsymbol{x}\|_{X}} .
$$

If $X=Y$ is a Hilbert space, then $\|\cdot\|_{2}$ denotes the operator norm defined by (48) for maps from $X$ to itself.

Define the functions

$$
\begin{aligned}
M_{f}(t) & =\sup _{s \geq t}\|\mathscr{F}(s)\|, \\
I_{f}(t) & =\int_{t}^{\infty}\|\mathscr{F}(s)\| d s .
\end{aligned}
$$

In these formulæ $\|\cdot\|$ is an operator norm on $2 \times 2$ matrices. We say that the (right) Marchenko equation can be solved by simple iteration on the interval $[t, \infty)$ if the sequence defined by

$$
\begin{aligned}
& \mathscr{K}_{t}^{0}(s)=-\mathscr{F}(s+t), \\
& \mathscr{K}_{t}^{j}(s)=-\mathscr{F}(s+t)-\int_{t}^{\infty} \mathscr{K}_{t}^{j-1}(x) \mathscr{F}(s+x) d x, \quad j=1,2, \ldots
\end{aligned}
$$

converges uniformly to $\mathscr{K}_{t}(s)$ for $s \in[t, \infty)$.

Theorem 5. If $I_{f}(2 t)<1$, then the Marchenko equation can be solved by simple iteration on the interval $[t, \infty)$. The solution satisfies the estimate

$$
\mathscr{K}_{t}(s) \leq \frac{M_{f}(2 t)}{1-I_{f}(2 t)} .
$$

The proof is given in the Appendix. Note that no assumption is made concerning the presence or absence of bound states.

For the minimum energy pulse there are no bound states. In all examples we have computed thus far, the minimum energy pulse and the first order approximation 
$\left(-2 f^{*}(2 t)\right)$ have essentially the same amplitude and effective support. This is in stark contrast to the pulses obtained in [14] using the method of Moses and Proesser. In Figure 1 we compare the minimum energy $90^{\circ}$ pulse (with a $20 \%$ transition window) to the first order approximation. This observation agrees with (45), which implies that the $L^{2}$-norm of $q$ is less than the $L^{2}$-norm of $-2 f^{*}(2 t)$.

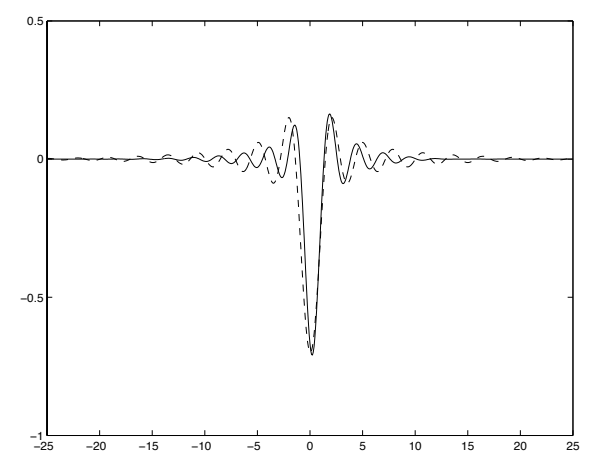

Figure 1. A comparison of the minimum energy $90^{\circ}$ pulse having a $20 \%$ transition zone (solid line) with the first order approximation (dashed line).

The estimate (51) implies that, so long as the hypotheses of the theorem hold, the potential $q$ satisfies the estimate

$$
|q(t)| \leq \frac{2 M_{f}(2 t)}{1-I_{f}(2 t)}
$$

This in turn explains our claim that the essential support of $q$ is determined by the essential support of $f$. A similar result holds for the left Marchenko equation. Taken together they show that if $r$ decays rapidly enough and is sufficiently smooth, then the potential $q$ can be determined, by simple iteration outside of a bounded interval $\left[t_{-}, t_{+}\right]$. Note that

$$
\|f\|_{L^{1}}=I_{f}(-\infty) .
$$

If $\|f\|_{L^{1}}<1$, then $q$ can be determined on the whole real line by iteration using just the right Marchenko equation. While it is by no means obvious from the definition of $g$, (46), we have found, empirically, that $\|f\|_{L^{1}} \approx\|g\|_{L^{1}}$. Moreover, we find that, if $\|f\|_{L^{1}}<2$, then $q$ can be found by using each Marchenko equation once.

If $I_{f}(t) \geq 1$ then we may need to modify the iteration to solve (40). Since $F_{t}^{*} F_{t}$ is a positive operator, it has purely positive spectrum. If the norm, $\left\|F_{t}^{*} F_{t}\right\|_{2}=\gamma$, then the spectrum of $F_{t}^{*} F_{t}$ lies in the interval $[0, \gamma]$ and therefore

$$
\left\|F_{t}^{*} F_{t}-\frac{\gamma}{2} \operatorname{Id}\right\|_{2} \leq \frac{\gamma}{2}
$$

We rewrite $(40)$ in the form

$$
\left[\mathrm{Id}+\frac{2}{2+\gamma}\left(F_{t}^{*} F_{t}-\frac{\gamma}{2} \mathrm{Id}\right)\right] \mathscr{K}_{12 t}=\frac{2}{2+\gamma} f^{*}(t+s) .
$$


As

$$
\left.\| \frac{2}{2+\gamma}\left(F_{t}^{*} F_{t}-\frac{\gamma}{2} \mathrm{Id}\right)\right) \|_{2}<\frac{\gamma}{2+\gamma},
$$

equation (53) can again be solved by a simple iteration. While, in practice, iteration seems to be fastest, numerical approximations to (40) can easily be solved using the conjugate gradient method or Cholesky factorization. Because $\left\|F_{t}^{*} F_{t}\right\|_{2}=\left\|F_{t}\right\|_{2}^{2}$, it follows easily from the Cauchy-Schwarz inequality that

$$
\left\|F_{t}^{*} F_{t}\right\|_{2} \leq=\left[\int_{2 t}^{\infty}|f(s)| d s\right]^{2} .
$$

Remark 11. After a potential $q$ with reflection coefficient $r$ and no bound states is found, it is a relatively simple matter to add in bound states. An algorithm to do this is described in section 6 of [3]. One possible usage for bound states is to reduce the effective support of the potential. To that end, one might chose points $\left\{\xi_{1}, \ldots, \xi_{N}\right\}$ in the upper half plane and norming constants $\left\{C_{j}\right\}$ so that sum,

$$
i \sum_{j=1}^{N} C_{j} e^{i \xi_{j} t}
$$

approximates

$$
\check{r}(t)=\frac{1}{2 \pi} \int_{-\infty}^{\infty} r(\xi) e^{i \xi t} d \xi
$$

over some ray $[T, \infty)$. With these choices the effective support of $f$ defined in (37) can be made smaller than that of $\check{r}$ and thereby a potential with smaller (effective) support, producing the same magnetization profile, can be found. This is of course at the expense of

$$
4 \sum_{j=1}^{N} \operatorname{Im} \xi_{j}
$$

in additional energy. In Section 6 we show that the effective support of $q$ determines the rephasing which is needed to obtain the specified magnetization profile.

While the bound states do not affect the observable magnetization, they do affect the spins themselves. Bound states might prove useful in designing pulse sequences intended to manipulate higher order quantum coherences.

\section{What Is Time?}

Before we turn to the computation of examples, we need to consider the physical significance of the $t$-parameter which appears in (11). This is intimately connected to the problem of rephasing. The main clue comes from (36). Suppose that $q$ is a potential 
supported in $\left[t_{0}, t_{1}\right]$ with reflection coefficient $r$. After time $t_{1}$, a solution to (11) is freely precessing in the local (gradient offset) $\boldsymbol{B}_{0}$-field.

If $t_{1}=0$, then, at the end of the RF-excitation, the magnetization satisfies

$$
\frac{\left(m_{11-}+i m_{21-}\right)(\xi ; 0)}{1+m_{31-}(\xi ; 0)}=r(\xi) .
$$

We have therefore achieved the desired magnetization profile without any need for rephasing. If on the other hand $t_{1}<0$, then, at the end of the excitation, the magnetization satisfies

$$
\frac{\left(m_{11-}+i m_{21-}\right)\left(\xi ; t_{1}\right)}{1+m_{31-}\left(\xi ; t_{1}\right)}=e^{2 i \xi t_{1}} r(\xi) .
$$

In order to get the desired magnetization profile the spins must freely precess for $\left|t_{1}\right|-$ units of time. Finally if $t_{1}>0$ then the spins must precess for $-t_{1}$-units of time. In other words, either a $180^{\circ}$-refocusing pulse must be applied, or the gradient needs to be reversed, followed by $t_{1}$-units of free precession, in order to achieve the magnetization profile specified by $r$.

In RF-pulse synthesis the data is specified in the frequency domain. Given $r(\xi)$ and perhaps some bound states $\left\{\left(\xi_{j}, C_{j}\right)\right\}$, the IST produces a potential which is a function of $t$. The time parameterization of this potential is determined by the reduced scattering data and thereby determines what sort of rephasing is needed to achieve the specified magnetization profile. If the essential support of the potential is contained in $\left[t_{0}, t_{1}\right]$, then, according to the sign of $t_{1}$, the necessary rephasing is determined as in the previous paragraph. The main point to note is that the time dependence of the potential is an output of the IST. In particular, the numerical value of $t_{1}$, in appropriate units, determines the rephasing which is needed to achieve the specified magnetization profile. The rephasing time is therefore an output of the IST method.

Two tools are available to manipulate the support of $q$ : (1) We can use smoother approximations to $r$ so that its Fourier transform is more rapidly decreasing (see Examples 1-3). The estimate (52) shows that this reduces the essential support of $q$. (2) The other tool is the introduction of bound states, coupled with methods of meromorphic approximation. Rourke and Morris approximate $r$ by rational functions which decay at infinity, and obtain potentials with support in $(-\infty, 0]$. The rational approximations to $r$ which they use have poles in the upper half plane, and these, in turn, lead to an increase in the energy and the oscillation of the RF-envelope. This is the price for an envelope that does not require rephasing. For the standard pulses used in MR imaging, the minimum energy pulse in general requires rephasing. When computing the total energy requirement for an RF-envelope with $t_{1}>0$, one must include the energy of the (hard) $180^{\circ}$-pulse or that of transients produced by reversing the gradient.

The problem of finding approximations that carefully balance the rephasing requirements with the energy of the pulse itself seems a very interesting field for further investigation. Through the Marchenko equations and the Paley-Wiener Theorem II, it translates to the problem of approximating a function $r$, defined on real axis, by meromorphic functions $\rho$, defined in the upper half plane, which satisfy an estimate of the 
form

$$
\int_{-\infty}^{\infty} e^{-4 \eta T}|\rho(\xi+i \eta)|^{2} d \xi \leq C \text { for } \eta \geq 0 .
$$

Here $C$ and $T$ are positive constants. The resultant potential will be supported in $(-\infty, T]$. We will return to this problem in a subsequent publication.

\section{Analysis of the Hard Pulse Approximation}

The Shinnar-Le Roux algorithm uses the hard pulse approximation to design an RFenvelope. Given a magnetization profile, $r(\xi)$, the SLR-algorithm designs a potential of the form

$$
q_{0}=\sum_{j=1}^{N} \mu_{j} \delta(t-j \Delta),
$$

to produce an approximation to the desired profile over a subinterval of

$$
\left[-\Delta^{-1} \pi, \Delta^{-1} \pi\right] .
$$

The reflection coefficient $r_{0}$, defined by $q_{0}$, is a periodic function of period $\Delta^{-1} 2 \pi$. The SLR-algorithm provides an iterative method for determining the coefficients $\left\{\mu_{j}\right\}$.

Of course, a sum of $\delta$-pulses is nonphysical, requiring infinite energy to realize. The RF-envelope which is actually used is a "softened" version of $q_{0}$. For example one could replace each $\mu_{j} \delta(t-j \Delta)$ by a boxcar pulse of width $\Delta$ with the same area, leading to the softened pulse:

$$
q_{1}=\sum_{j=1}^{N} \frac{\mu_{j}}{\Delta} \chi_{[0, \Delta)}(t-j \Delta) ;
$$

see [16]. The reflection coefficient $r_{1}$ corresponding to $q_{1}$ tends to zero at infinity. There is no reasonable sense in which the difference $q_{0}-q_{1}$ is "small." Nonetheless, under certain conditions, the difference $\left|r_{0}(\xi)-r_{1}(\xi)\right|$ can be made small for $\xi$ in a fixed interval. The relationship between $r_{0}$ and $r_{1}$ is studied below.

The SLR approach should be contrasted with the approach outlined above: Given $r$ we approximate it by a smooth magnetization profile $r_{s}$. We then construct a smooth potential $q_{s}$ with reflection coefficient $r_{s}$. Such a pulse is again implemented by replacing it with a sum like $q_{1}$. So there should be no confusion, we denote a sequence of boxcar pulses approximating $q_{s}$ by $q_{s}$. Below we show that the difference in the magnetization profile produced by $q_{s}$, and that produced by $q_{s 1}$ is controlled by $\left\|q_{s 1}-q_{s}\right\|_{L^{1}}$.

For our analysis of the hard pulse approximation we consider the one parameter family of pulses

$$
q_{\epsilon}=\sum_{j=1}^{N} \frac{\mu_{j}}{\epsilon \Delta} \chi_{[0, \epsilon \Delta)}(t-j \Delta), \quad \epsilon \in[0,1],
$$


with the understanding that $q_{0}$ is the sum of $\delta$-pulses given above. It is a simple computation to show that

$$
\int_{-\infty}^{\infty}\left|q_{\epsilon}(t)\right| d t
$$

is independent of $\epsilon$, and

$$
\int_{-\infty}^{\infty}\left|q_{\epsilon}(t)\right|^{2} d t=\frac{1}{\epsilon} \int_{-\infty}^{\infty}\left|q_{1}(t)\right|^{2} d t
$$

This formula indicates the desirability of using $q_{1}$ to approximate $q_{0}$, rather than $q_{\epsilon}$ for an $\epsilon<1$.

Let $r_{\epsilon}$ denote the reflection coefficient defined by $q_{\epsilon}$. The potential $q_{\epsilon}$ is supported in the interval $[0, N \Delta]$. In order to study $r_{\epsilon}$ we need to find a formula for $\psi_{\epsilon 1-}(\xi ; N \Delta)$. As is well known, this can be conveniently expressed in terms of a product of $2 \times 2$ matrices. The solution operator at time $t_{0}+\Delta$ to the ZS-system with potential given by

$$
\epsilon^{-1} \mu \chi[0, \epsilon \Delta)\left(t-t_{0}\right)
$$

is

$$
U_{\mu, \epsilon, \Delta}(\xi)=\left[\begin{array}{cc}
e^{-i(1-\epsilon) \xi \Delta}\left(\frac{\alpha \cos (\alpha)-i \epsilon \xi \Delta \sin (\alpha)}{\alpha}\right) & e^{-i(1-\epsilon) \xi \Delta}\left(\frac{\mu \sin (\alpha)}{\alpha}\right) \\
-e^{i(1-\epsilon) \xi \Delta}\left(\frac{\mu^{*} \sin (\alpha)}{\alpha}\right) & e^{i(1-\epsilon) \xi \Delta}\left(\frac{\alpha \cos (\alpha)+i \epsilon \xi \Delta \sin (\alpha)}{\alpha}\right)
\end{array}\right]
$$

where

$$
\alpha=\sqrt{(\epsilon \xi \Delta)^{2}+|\mu|^{2}} .
$$

The solution operator for the ZS-system, at time $N \Delta$ with the potential given by (58) is therefore the product of the $2 \times 2$-matrices:

$$
\begin{aligned}
U_{\epsilon, \Delta}(\xi) & =U_{\mu_{N}, \epsilon, \Delta}(\xi) \cdots U_{\mu_{1}, \epsilon, \Delta}(\xi) \\
& =\left[\begin{array}{cc}
A_{\epsilon}(\xi) & -B_{\epsilon}^{*}(\xi) \\
B_{\epsilon}(\xi) & A_{\epsilon}^{*}(\xi)
\end{array}\right] .
\end{aligned}
$$

It follows easily from (35) that

$$
r_{\epsilon}(\xi)=\frac{B_{\epsilon}(\xi) e^{-2 i N \xi \Delta}}{A_{\epsilon}(\xi)}
$$

The entries of (59), and (61) are continuous at $\epsilon=0$. The functions

$$
\left(e^{i N \xi \Delta} A_{\epsilon}, e^{-i N \xi \Delta} B_{\epsilon}\right)
$$

are none other than the scattering coefficients for the ZS-system with potential $q_{\epsilon}$. If $\epsilon=0$ then $A_{0}$ and $B_{0}$ can be expressed as

$$
A_{0}(\xi)=e^{i N \xi \Delta} P\left(e^{i \xi \Delta}\right), \quad B_{0}(\xi)=e^{-i N \xi \Delta} Q\left(e^{i \xi \Delta}\right),
$$


Where $P$ and $Q$ are polynomials of degree $N-1$. As expected $A_{0}$ and $B_{0}$ are $\Delta^{-1} 2 \pi$ periodic functions.

The SLR design process begins with $r_{0}(\xi)$, a rational function of an appropriate degree in $e^{i \xi \Delta}$, that approximates the desired magnetization profile, $r(\xi)$, over the interval $\left[-\Delta^{-1} \pi, \Delta^{-1} \pi\right]$. The algorithm then determines coefficients $\left\{\mu_{j}\right\}$ so that

$$
r_{0}(\xi) \approx \frac{B_{0}(\xi) e^{-2 i N \xi \Delta}}{A_{0}(\xi)} .
$$

As with the IST approach, this problem does not have a unique solution. The different solutions are labeled, in essence, by the locations of the zeros of $P$ outside the unit disk. These correspond to zeros of $A_{0}$ in the upper half plane.

In fact, we note that the SLR-method is a special case of the inverse scattering approach, wherein the potentials under consideration are restricted to have the form given in (57). For potentials of this special form (and rational, periodic approximations to $r$ ) the SLR-algorithm provides a direct method for inverting the scattering transform, similar to the method of Moses and Proesser.

The questions of principal interest are:

1. How well does $r_{1}(\xi)$ approximate $r_{0}(\xi)$ over the interval $\left[-\Delta^{-1} \pi, \Delta^{-1} \pi\right]$ ?

2. How rapidly does $r_{1}$ decay outside of $\left[-\Delta^{-1} \pi, \Delta^{-1} \pi\right]$ ?

3. How are the zeros of $A_{1}$ in the upper half plane related to those of $A_{0}$ ?

The first question admits of a fairly simple analysis. We first consider a single term in the product expansion (61), examining the dependence of the difference $\left\|U_{\mu, 1, \Delta}(\xi)-U_{\mu, 0, \Delta}(\xi)\right\|$ on $(\mu, \Delta)$, for $\xi$ in the interval $\left[-\Delta^{-1} \pi, \Delta^{-1} \pi\right]$. By setting $\zeta=\xi \Delta$ this reduces to consideration of the differences

$$
\begin{aligned}
& D_{1}(\mu, \zeta)=\left|e^{i \zeta}\left(\cos \sqrt{|\mu|^{2}+\zeta^{2}}-\frac{i \zeta \sin \sqrt{|\mu|^{2}+\zeta^{2}}}{\sqrt{|\mu|^{2}+\zeta^{2}}}\right)-\cos \right| \mu|| \\
& D_{2}(\mu, \zeta)=\left|e^{i \zeta} \frac{\mu \sin \sqrt{|\mu|^{2}+\zeta^{2}}}{\sqrt{|\mu|^{2}+\zeta^{2}}}-\frac{\mu \sin |\mu|}{|\mu|}\right|
\end{aligned}
$$

for $\zeta \in[-\pi, \pi]$. As the plots in Figures 2 and 3 show, the only way to make both differences small is to take $|\mu|$ small.

In terms of pulse design, this means that $\Delta$ should be taken to be small, which in turn forces $N$ to be large. A computation with Taylor series shows that

$$
\begin{aligned}
& D_{1}(\mu, \zeta)=|\mu|^{2} F(|\mu|, \zeta), \\
& D_{2}(\mu, \zeta)=|\mu \zeta| G(|\mu|, \zeta),
\end{aligned}
$$

where $F$ and $G$ are entire functions. For a fixed target magnetization $r$, the $\left\{\mu_{j}\right\}$ appearing in (61) satisfy

$$
\left|\mu_{j}\right|=O(\Delta)=O\left(N^{-1}\right) .
$$




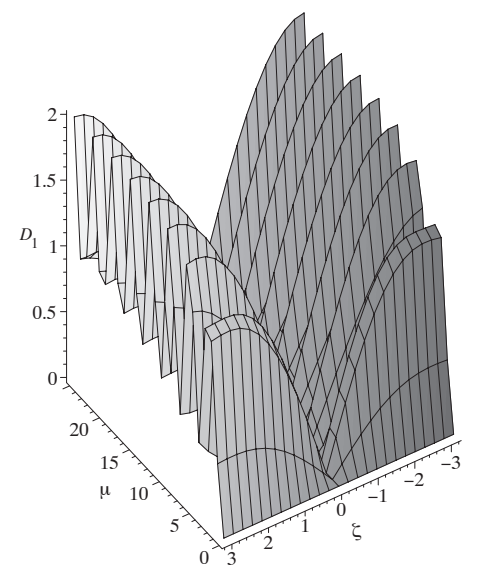

(a) A plot of $D_{1}$.

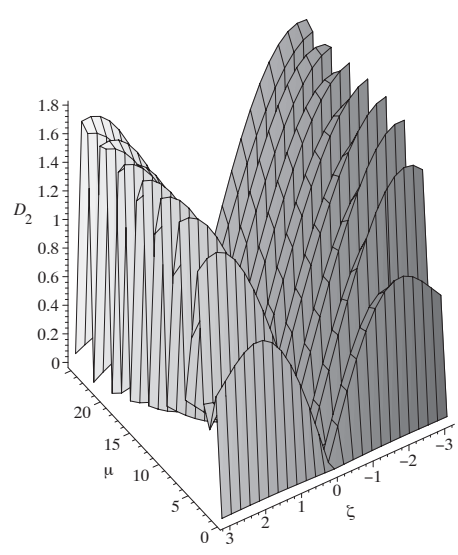

(b) A plot of $D_{2}$.

Figure 2. Plots of the error functions $D_{1}$ and $D_{2}$ for $\mu \in[0,25]$.

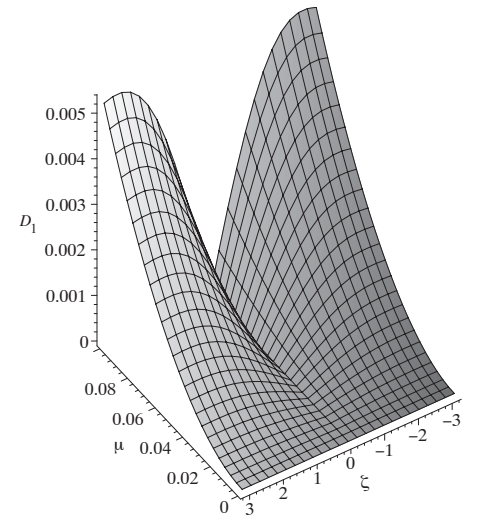

(a) A plot of $D_{1}$.

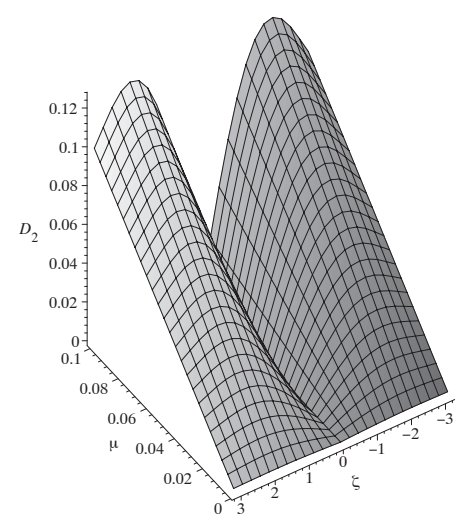

(b) A plot of $D_{2}$.

Figure 3. Plots of the error functions $D_{1}$ and $D_{2}$ for $\mu \in[0,0.1]$. 
For $\xi$ restricted to a fixed finite interval, $\zeta$ also behaves like $O\left(N^{-1}\right)$ as $N \rightarrow \infty$. With this restriction on $\xi$, both error functions behave like $O\left(N^{-2}\right)$ as $N$ tends to infinity.

We express $U_{1, \Delta}$ as

$$
U_{1, \Delta}=U_{\mu_{N}, 0, \Delta}\left(\operatorname{Id}_{2}+E_{N}\right) U_{\mu_{N-1}, 0, \Delta}\left(\operatorname{Id}_{2}+E_{N-1}\right) \cdots U_{\mu_{1}, 0, \Delta}\left(\operatorname{Id}_{2}+E_{1}\right),
$$

where

$$
\mathrm{Id}_{2}+E_{j}=U_{\mu_{j}, 0, \Delta}^{-1} U_{\mu_{j}, 1, \Delta} .
$$

Using this expression and the estimates above one can show that the differences

$$
\left|A_{1}(\xi)-A_{0}(\xi)\right|, \quad\left|B_{1}(\xi)-B_{0}(\xi)\right|
$$

can be made uniformly $O\left(N^{-1}\right)$ for $\xi$ belonging to a fixed interval, provided $N$ is sufficiently large. (If the error in the individual terms were of order $N^{-1}$, then this statement would not be true. This is indicated by the well known formula $\lim _{N \rightarrow \infty}\left(1+\frac{1}{N}\right)^{N}=$ $e$.) Thus by taking $N$ large and therefore $\Delta$, small the softened SLR pulse should provide a good approximation to the desired magnetization over a fixed interval where $A_{0}$ does not vanish.

Empirically, it is well known that $r_{1}$ is quite small outside of $\left[-\Delta^{-1} \pi, \Delta^{-1} \pi\right]$. For $\xi \Delta$ large, the diagonal terms of $U_{\mu, 1, \Delta}(\xi)$ are well approximated by $e^{ \pm i \xi \Delta}$, while the off-diagonal terms behave like $\mu \operatorname{sinc}(\xi \Delta),\left(-\mu^{*} \operatorname{sinc}(\xi \Delta)\right.$, respectively). For large $\xi, U_{1, \Delta}(\xi)$ therefore has decaying side lobes, with periodicity $\frac{2 \pi}{\Delta}$. This is apparent in Figure 4(c), which shows the magnitude of the magnetization profiles for several soft pulse approximations to a $90^{\circ}$-sinc-pulse with a bandwidth of $1000 \mathrm{~Hz}$. Note that $r_{\frac{1}{2}}$ has considerable support outside the fundamental period $[-500,500]$. These side lobes appear whenever the soft pulse approximation is used.

The answer to the last question is not immediately clear. According to [13], the minimum energy SLR pulse is attained by placing all the roots of $P$ inside the unit circle. This accords well with our analysis, as it is zeros of $a$ in the upper half plane (which corresponds to the exterior of the unit circle) that increase the energy requirements of an RF-envelope. The asymptotic analysis above shows that zeros of $A_{0}$ in the upper half plane, that are close to the real segment $\left[-\Delta^{-1} \pi, \Delta^{-1} \pi\right]$, are likely to produce zeros of $A_{1}$ in the upper half plane, thereby increasing the energy requirement of the softened pulse. The behavior of the zeros of $A_{\epsilon}$, as $\epsilon$ goes from 0 to 1 , seems an interesting question for further investigation.

By way of contrast, using the IST method, one is in direct control of the zeros of $a$ in the upper half plane as well as the phase of the magnetization profile. We close this section by stating an estimate which shows that replacing the smooth RF-envelope, found using the IST, by a soft pulse approximation $q_{s 1}$ does not result in substantial errors in the magnetization profile.

Theorem 6. Suppose that $h_{1}$ and $h_{2}$ are integrable potentials for the ZS-system, and 


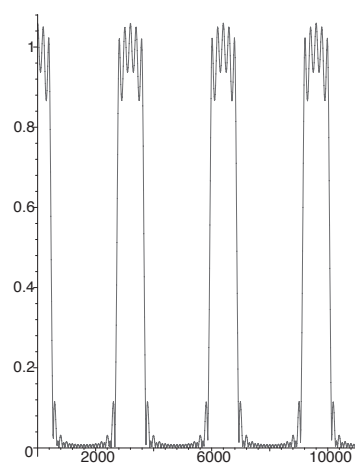

(a) A plot of $\left|r_{0}\right|$, the hard pulse approximation.

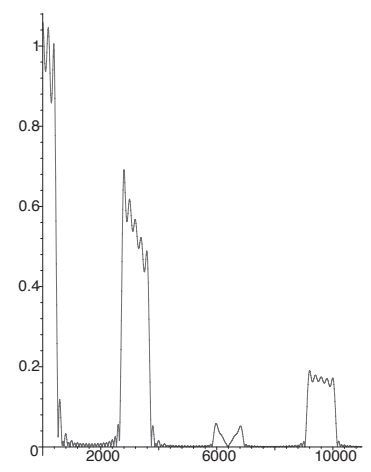

(b) A plot of $\left|r_{\frac{1}{2}}\right|$, an intermediate case.

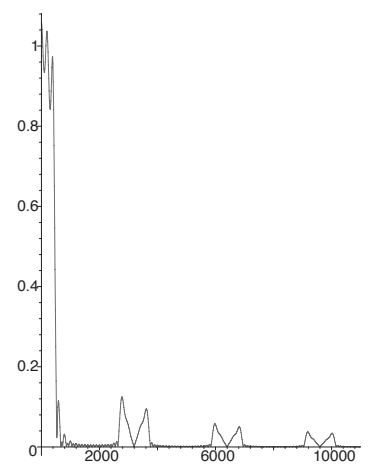

(c) A plot of $\left|r_{1}\right|$, the soft pulse approximation.

Figure 4. Plots of the absolute magnetization profiles for various approximations to a $90^{\circ}$-sinc pulse showing several fundamental periods.

that $F_{i}^{ \pm}(\xi), i=1,2$ solves the ZS-system with potential $h_{i}, i=1,2$. Let

$$
\begin{array}{ll}
H^{-}(t)=\int_{-\infty}^{t}\left|h_{1}(s)-h_{2}(s)\right| d s, & Q^{-}(t)=\int_{-\infty}^{t}\left|h_{1}(s)\right| d s, \\
H^{+}(t)=\int_{t}^{\infty}\left|h_{1}(s)-h_{2}(s)\right| d s, & Q^{+}(t)=\int_{t}^{\infty}\left|h_{1}(s)\right| d s .
\end{array}
$$

If

$$
\lim _{t \rightarrow \pm \infty}\left\|F_{1}^{ \pm}(\xi ; t)-F_{2}^{ \pm}(\xi ; t)\right\|=0 .
$$

Then, for $\xi \in \mathbb{R}$ the differences satisfy the estimates

$$
\left\|F_{1}^{ \pm}(\xi ; t)-F_{2}^{ \pm}(\xi ; t)\right\| \leq 2 I_{0}\left(2\left\|h_{2}\right\|_{L^{1}}\right) H^{ \pm}(t) \exp \left(Q^{ \pm}(t)\right) .
$$

The proof is in the Appendix.

In light of these estimates, it is clear that, by choosing a soft approximation, $q_{s}$, to the smooth pulse, $q_{s}$, so that $\left\|q_{s}-q_{s 1}\right\|_{L^{1}}$ is small, the reflection coefficient $r_{s 1}$ can be made uniformly close to $r_{s}$ over any desired interval where $a_{s}$ does not vanish. Once again, this amounts to using a small value of $\Delta$ in the definition of $q_{s 1}$. To assure that the energy in $q_{s 1}$ is close to that in $q_{s}$ requires the $L^{2}$-error, $\left\|q_{s}-q_{s 1}\right\|_{L^{2}}$, to be small. On the one hand,

$$
\left\|q_{s}-q_{s 1}\right\|_{L^{2}} \leq \sqrt{\left\|q_{s}-q_{s 1}\right\|_{L^{\infty}}\left\|q_{s}-q_{s 1}\right\|_{L^{1}}}
$$


while, on the other hand, if $q_{s}$ and $q_{s 1}$ are supported in an interval of length $l$, then

$$
\left\|q_{s}-q_{s 1}\right\|_{L^{1}} \leq \sqrt{l}\left\|q_{s}-q_{s 1}\right\|_{L^{2}} .
$$

Thus both the $L^{1}$ and $L^{2}$ norms can be made small simultaneously.

\section{Examples}

We now present several examples of minimum energy pulses designed using the inverse scattering transform, as implemented via the Marchenko equations. We only work with data for which $\|f\|_{L^{1}} \leq 2.5$. The RF-envelopes were obtained by solving the left and right Marchenko equations using simple iteration. We will return to the numerical issues connected with solving the Marchenko equation for large data in a later publication. The examples in this section were computed using a MATLAB ${ }^{\circledR}$ program, with no serious attempt made to optimize the speed of the computations. Each pulse took between 5 and 15 minutes to construct; it is likely that this can be considerably reduced. The computation time grows a bit slower than $N^{3}$, with $N$ the number of points where $q$ is determined.

In the examples below the potentials are computed at 1024 points lying in the time interval [ $-40,40]$ (arbitrary units); the time step is $d t=0.0785$. The horizontal axes, in the graphs of the potentials, are in arbitrary time units, and the unit along vertical axis is $\frac{1}{2}$-radians/time-unit. Recall that the RF-envelope, $\omega$, is $-2 i \gamma^{-1} q^{*}$. We also plot the stereographic magnetization profiles, $r_{s}(\xi)$, produced by the soft pulse approximation to $q$, defined by

$$
q_{s}(t)=\sum_{j=J_{\min }}^{J_{\max }} q_{c}(j d t) \chi_{[0, d t)}(t-j d t) .
$$

Here $\left\{q_{c}(j d t): J_{\min } \leq j \leq J_{\max }\right\}$ are the computed values of $q$ lying in its effective support.

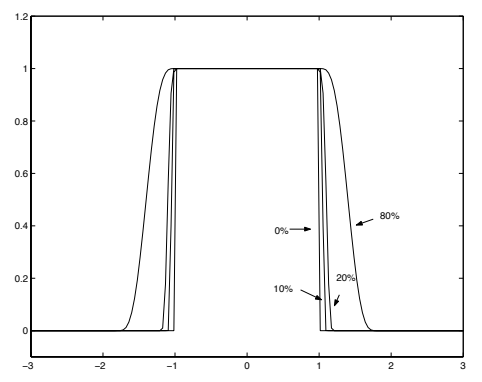

Figure 5. Regularized windows used in the approximation of $90^{\circ}$ pulses

We give plots of the real and imaginary parts of $r_{s}(\xi)$, for $\xi$ lying in a small interval around the support of $r$. But for rapidly decaying side lobes produced by the soft pulse approximation, occurring at intervals of $2 \pi / d t$, (see Figure 4) $r_{s}$ is very small outside 
the interval shown in the graphs. In these plots the variable $\xi$, lying along the horizontal axis, has units of $\frac{1}{2}$-radians/time-unit, the vertical axis is dimensionless. In most of our examples $r$ is real valued, so the phase error is represented by the imaginary part of $r_{s}$. From (36) we see that $0 \leq|r|<1$ corresponds to flip angles less than $90^{\circ} ;|r|=1$ corresponds to a flip angle of $90^{\circ} ; 1<|r|<\infty$ correspond to flip angles greater than $90^{\circ}$. Finally, $|r|=\infty$ corresponds to a flip angle of $180^{\circ}$. The magnetization is rephased essentially according to the prescription in section 6 . Because we are working with a discretization of a continuum model, some interpretation is required. In most examples, we actually rephase for $.45 d t$ time units less than the upper limit of the theoretical effective support of the potential.

For the graphs in this section, the real part of a function is shown with a solid line and the imaginary part with a dashed line.

\section{1 $90^{\circ}$ Pulses}

$90^{\circ}$-pulses with a variety of regularizations. We consider the design of $90^{\circ}$-pulses. For these examples, the "ideal" profile, $r(\xi)=\chi_{[-1,1]}(\xi)$, is approximated by piecewise polynomial functions with three continuous derivatives. We obtain pulses with $10 \%, 20 \%$, and $80 \%$ windows. The profiles used are shown in Figure 5. This collection of examples demonstrates the close connection between the smoothness of $r$ and the effective support of $q$.

Example 1 (10\% window). Our first approximation to a $90^{\circ}$ pulse has a very short transition region. The sharp transitions produce a slowly decaying pulse. While it is well rephased away from the corners, it displays considerable oscillation near the transitions.

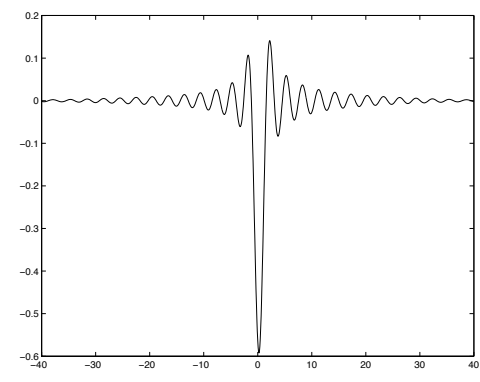

(a) The minimum energy potential

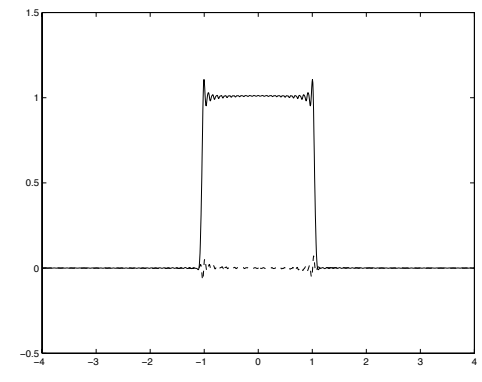

(b) The corresponding stereographic magnetization profile

Figure 6. The minimum energy potential and magnetization profile for an approximation to a $90^{\circ}$ pulse with a $10 \%$ transition zone.

Example 2 (20\% window). Our next approximation to a $90^{\circ}$ pulse has a somewhat larger transition region. The pulse has about half the effective support. The corre- 
sponding magnetization profile is very well rephased, with only the slightest ripple near points of finite differentiability.

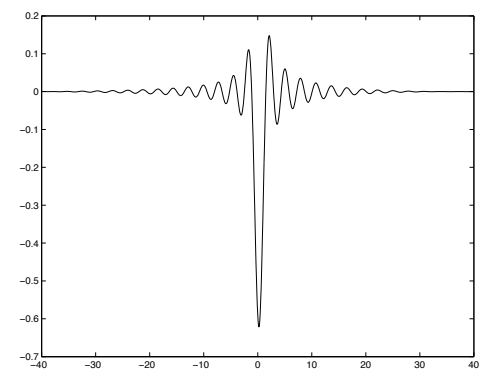

(a) The minimum energy potential

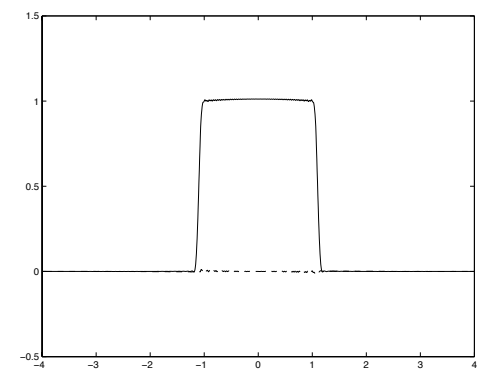

(b) The corresponding stereographic magnetization profile

Figure 7. The minimum energy potential and magnetization profile for an approximation to a $90^{\circ}$ pulse with a $20 \%$ transition zone.

Example 3 (80\% window). Our last approximation to a $90^{\circ}$ pulse has a transition region whose total length equals $1.6=.8 \times 2$. This pulse has much smaller effective support. The corresponding magnetization profile is almost indistinguishable from the specified $r$.

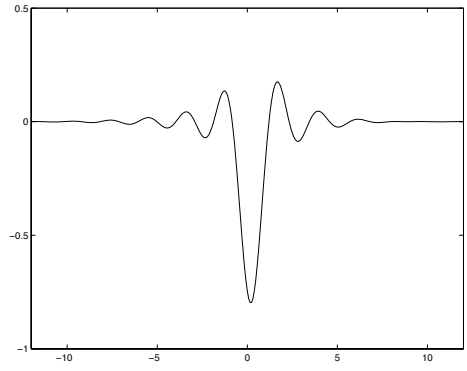

(a) The potential for a $90^{\circ}$

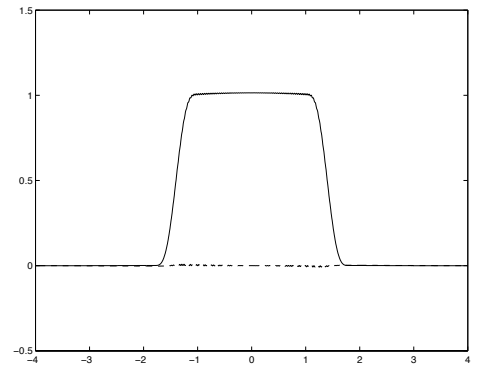

(b) The corresponding stereographic magnetization profile

Figure 8. The minimum energy potential and magnetization profile for an approximation to a $90^{\circ}$ pulse with a $80 \%$ transition zone.

Example 4 (Sensitivity to amplitude errors). In Figure 9 we show the magnetization profiles that result from using the potential in Example 2 scaled by .9 and 1.1, respectively. These pulses were rephased exactly as in Example 2. 


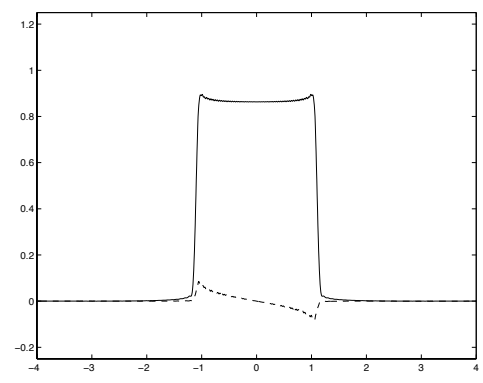

(a) $90 \%$

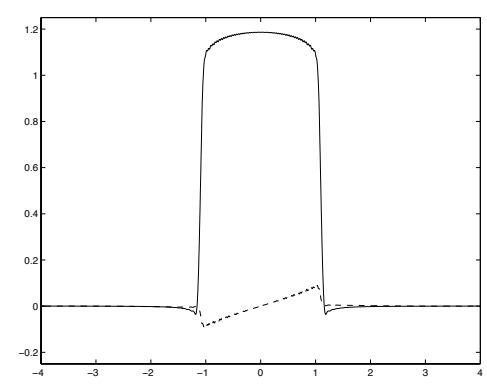

(b) $110 \%$

Figure 9. The consequences of errors in the amplitude for a $90^{\circ}$ pulse with a $20 \%$ transition region.

Figure 10(a) shows the result of rephasing the 90\%-pulse for $.3 d t$ time units less than in Figure 9(a), while in Figure 10(b) we rephased the $110 \%$ pulse for $.45 d t$ time units more than in Figure 9(b). This indicates the possibility of using slightly nonlinear gradients, matched to the inhomogeneities in the $\boldsymbol{B}_{1}$-field, to more accurately rephase selective pulses across the whole field of view.

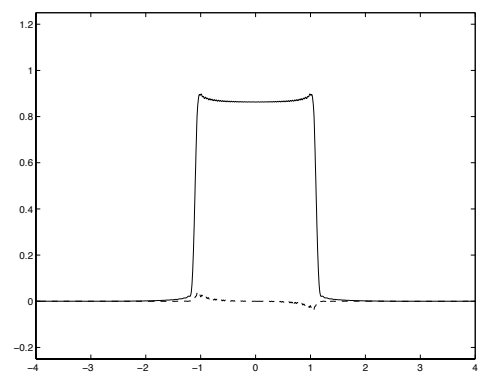

(a) $90 \%$

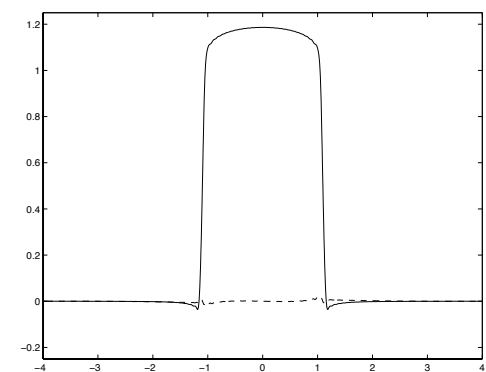

(b) $110 \%$

Figure 10. The consequences of errors in the amplitude for a $90^{\circ}$ pulse with a $20 \%$ transition region, with corrected rephasing.

Example 5 (The effects of truncation). In this example we examine the effects of truncating the pulse used in example 2 with a Gaussian window and a $\cos ^{2}$ window. The rephasing was, again, slightly adjusted to minimize the phase error near the center of the window. 


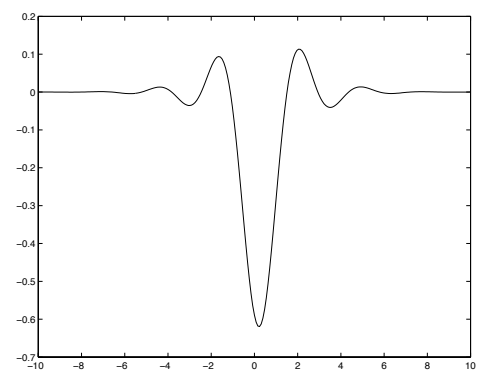

(a) Windowed pulse

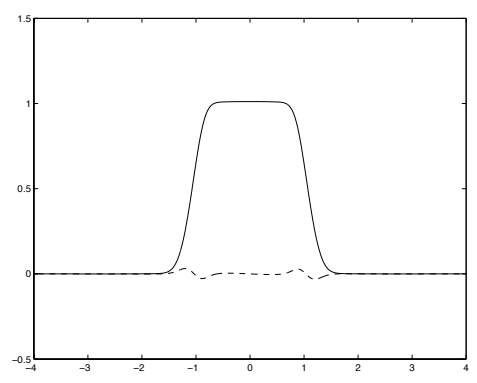

(b) Magnetization profile

Figure 11. The consequences of truncating the $20 \%$ window, $90^{\circ}$ pulse with a Gaussian window and corrected rephasing.

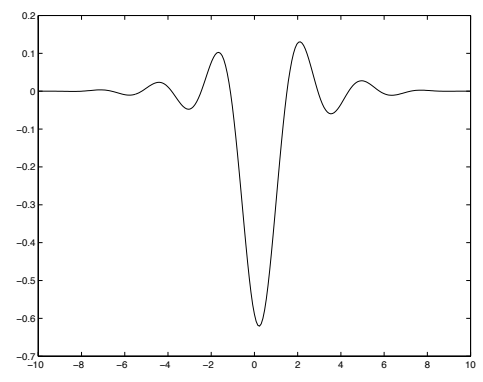

(a) Windowed pulse

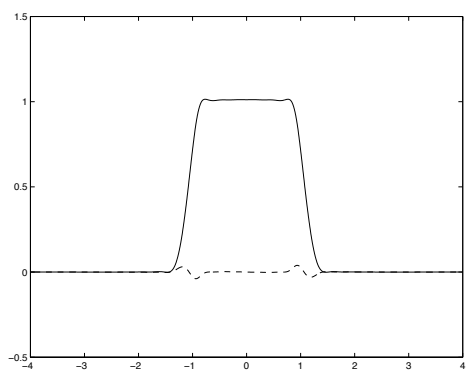

(b) Magnetization profile

Figure 12. The consequences of truncating the $20 \%$ window, $90^{\circ}$ pulse with a $\cos ^{2}$ window, with corrected rephasing.

It is notable that while these pulses are comparable in duration to the pulse in example 3 and their magnetization profiles have essentially equal widths, the pulse in example 3 produces virtually no phase error or overshoot.

\subsection{Two band pulses}

In these two examples we design minimum energy pulses which flip the magnetization $90^{\circ}$ in two disjoint frequency bands. In the first example the magnetization is flipped to the $x$-axis in both bands, while in the second, the magnetization is on the $x$-axis in the first band and the $y$-axis in the second. In both cases a $40 \%$ transition region is used. In the table at the end of this section we see that, as expected, the $L^{2}$-norms of these two profiles are essentially equal $(0.520$ versus 0.524$)$. 


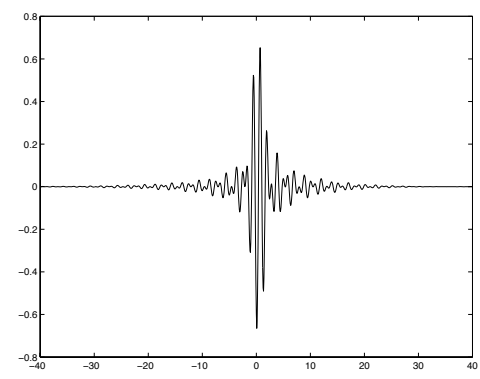

(a) Minimum energy pulse pulse

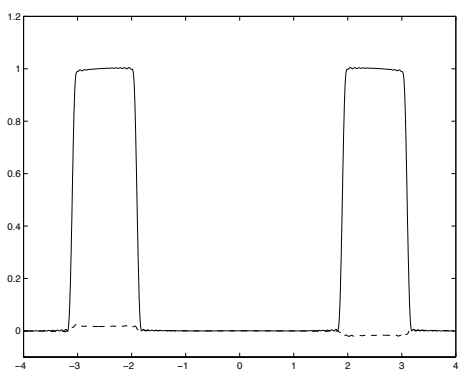

(b) Magnetization profile

Figure 13. Two disjoint bands, with magnetization in phase.

Example 6 (Two bands, in phase). Example 3 in [14] is designed to have the magnetization profile shown in Figure 13(b). If we interpret the time unit in this example as milliseconds, then $\omega(t)=5 i \gamma^{-1} q^{*}(2.5 t)$ would produce the magnetization profile in the cited example (our definition of $\omega$ is slightly different from that in [14]). The maximum amplitude of our scaled pulse is $3.3245 \mathrm{rad} / \mathrm{ms}$, which should be compared to a maximum amplitude of $\sim 50 \mathrm{rad} / \mathrm{ms}$ for the Rourke-Morris pulse. Our pulse has a duration of about $16 \mathrm{~ms}$ and requires about $8 \mathrm{~ms}$ of rephasing, whereas the RourkeMorris pulse has a duration of about $16 \mathrm{~ms}$ and does not require rephasing. The much larger amplitude and energy of the Rourke-Morris pulse is a consequence of the fact that the potential they construct has a large number of bound states.

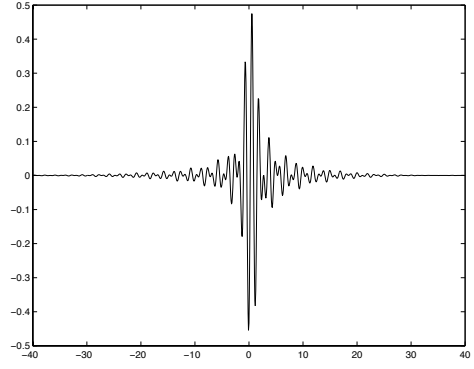

(a) Real part of the minimum energy pulse pulse

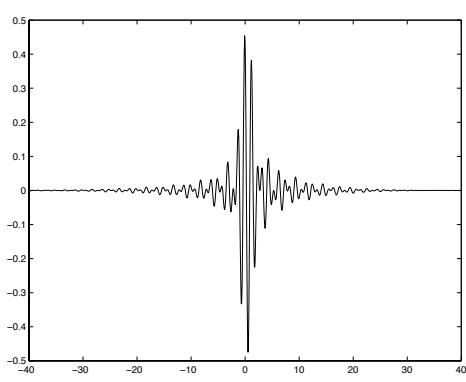

(b) Imaginary part of the minimum energy pulse pulse

Figure 14. The real and imaginary parts of the minimum energy pulse producing two disjoint bands, $90^{\circ}$ out of phase.

Example 7 (Two bands, $90^{\circ}$ out of phase). The profile is no longer symmetric, so the the pulse has nontrivial real and imaginary parts which are shown in Figure 14; the 


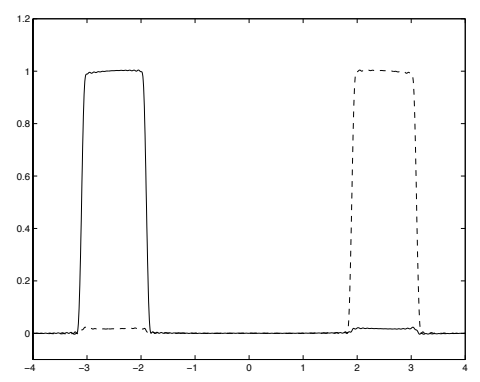

Figure 15. The magnetization profile with two disjoint bands, with magnetization $90^{\circ}$ out of phase.

corresponding magnetization profile is shown in Figure 15. The energy for this pulse is essentially identical to that in Example 6.

\subsection{Wavelet examples}

For our final examples we consider using standard wavelets to define the $x$-magnetization. Such pulses were earlier constructed by several investigators using the SLR method; for a discussion and references see [12]. If $\psi$ is a wavelet, then in the next two examples we set

$$
m_{1}(\xi)=\psi(\xi), m_{2}(\xi)=0, m_{3}(\xi)=\sqrt{1-\psi^{2}}
$$

Example 8 (Mexican hat). The Mexican hat wavelet is defined by the function

$$
\psi(\xi)=\frac{4}{\sqrt{3 \pi}}\left(1-\xi^{2}\right) e^{-\frac{\xi^{2}}{2}} .
$$

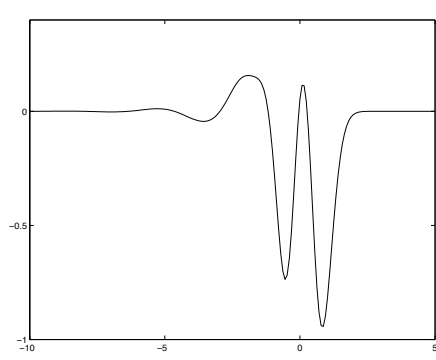

(a) Minimum energy pulse pulse

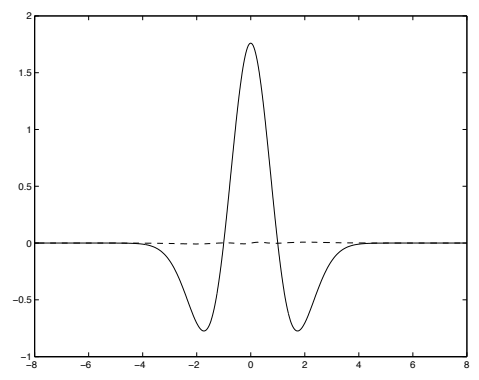

(b) Magnetization profile

Figure 16. The Mexican hat wavelet. 
Example 9 (Meyer wavelet). The Meyer wavelet is defined in the frequency domain by a rather complicated function with bounded support, for details see [7]. The real and imaginary parts of the minimum energy potential, as well as the magnetization profile, are shown in Figure 17.

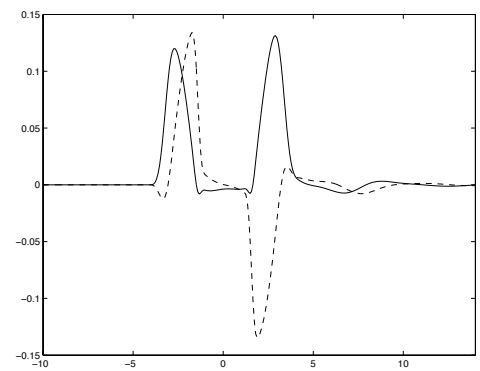

(a) Minimum energy pulse pulse

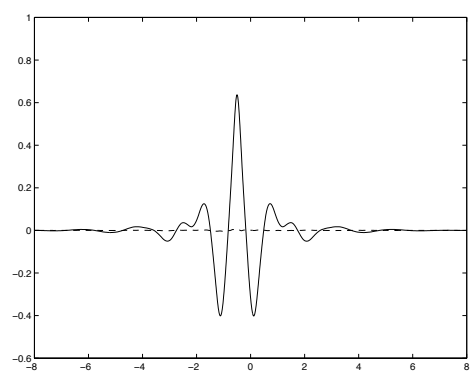

(b) Magnetization profile

Figure 17. The Meyer wavelet.

\subsection{Power computations}

In this final subsection we give a table of various numerical parameters connected to the RF-envelopes presented above. The first column contains the number of the figure depicting the potential, the second column is the energy for the given magnetization profile predicted by equation (42), and the third column is the $L^{2}$-norm of the computed RF-envelope. Though they are always a little larger, the computed values are very close to the predicted value (within 1.5\%). The actual magnetization profiles produced by the computed soft pulse approximations have decaying side lobes which account for the additional energy in column 3. The fourth column is the $L^{1}$-norm of $f$ defined in (37) and the fifth column is the $L^{1}$-norm of $g$ defined in (46). It is interesting to note that these numbers are nearly equal. The map from $f$ to $g$ preserves the $L^{2}$-norm, but there is no obvious reason why it should preserve the $L^{1}$-norm. It is also interesting that, for several of the pulses, $\|f\|_{L^{1}}$ and $\|g\|_{L^{1}}$ both exceed 2. Nonetheless we were able to find these pulses using only simple iteration.

\section{Conclusion}

Using the formula of Zakharov and Manakov, and the inverse scattering method we determine the minimum energy RF-envelope which produces a given magnetization profile. The minimum energy profile is the one for which there are no $L^{2}$-bound states. We show how the Marchenko equation can, in principle, be used to find this RF-envelope, and give an algorithm for solving it. The inverse scattering method also provides a corrected linear theory to replace the classical Fourier method. The minimum energy 


\begin{tabular}{|c|c|c|c|c|}
\hline Figure no. & $\frac{1}{\pi} \int \log \left(1+|r|^{2}\right)$ & $\int\left|q_{c}\right|^{2}$ & $\int|f|$ & $\int|g|$ \\
\hline \hline Fig. 6 & 0.4601 & 0.467 & 2.3328 & 2.3511 \\
\hline Fig. 7 & 0.4794 & 0.4850 & 2.1287 & 2.1734 \\
\hline Fig. 8 & 0.5950 & 0.6024 & 1.666 & 1.7081 \\
\hline Fig. 13 & 0.5175 & 0.520 & 2.39 & 2.4559 \\
\hline Fig. 14 & 0.5175 & 0.524 & 2.3907 & 2.4502 \\
\hline Fig. 16 & 0.1001 & 0.1002 & 0.5791 & 0.5791 \\
\hline Fig. 17 & 0.0626 & 0.0626 & 0.6429 & 0.6418 \\
\hline
\end{tabular}

Table 1. Energies and $L^{1}$-norms related to the computation of the examples above

RF-envelope is shown to have less energy than the corrected Fourier approximation. The minimum energy RF-envelope is computed in several representative examples. These pulses are seen to have much smaller amplitude and energy than those obtained by previous authors using the IST approach.

We also considered the SLR-method, showing that it is, in fact, a special (singular) case of the IST approach. We analyzed the nature of the approximations used in the SLR method, obtaining a criterion for the SLR RF-envelope to provide an accurate approximation to the desired magnetization profile over a specified band of offset frequencies. Because the approximations used in the (traditional) IST approach are much less singular, it would seem to provide a more incisive and broadly applicable tool for RF pulse sequence design than SLR. For example, one no longer needs to guess about the rephasing required by a RF-envelope; it is an output of the IST algorithm. Estimates for the decay of the RF-envelope are obtained in terms of the size of the first order approximate solution to the Marchenko equation. We give a practical algorithm for solving the Marchenko equation.

\section{Appendix: Mathematical Proofs}

The Paley-Wiener Theorems: The "Paley-Wiener" Theorem is used several times in this paper. This is really a collection of results relating the support properties of a function, defined on $\mathbb{R}$, to the analytic continuation of its Fourier transform. A complete discussion can be found in [19]. Two results adequate for our applications are:

Theorem 7 (Paley-Wiener Theorem I). A function $f$ in $L^{2}(\mathbb{R})$ has bounded support in the interval $[-L, L]$ if and only if its Fourier transform has an analytic extension to the entire complex plane such that $\hat{f}(\cdot+\eta) \in L^{2}(\mathbb{R})$, for all $\eta$, and

$$
|\hat{f}(\xi+i \eta)| \leq C e^{L|\eta|},
$$

for a constant $C$.

Theorem 8 (Paley-Wiener Theorem II). A function $f$ in $L^{2}(\mathbb{R})$ has support in the ray $(-\infty, 0]$ if and only if its Fourier transform has an analytic extension to the upper 
half plane satisfying

$$
\begin{gathered}
\int_{-\infty}^{\infty}|\hat{f}(\xi+i \eta)|^{2} \leq C \text { and } \\
\lim _{\eta \downarrow 0} \int_{-\infty}^{\infty}|\hat{f}(\xi+i \eta)-\hat{f}(\xi)|^{2}=0 .
\end{gathered}
$$

The proof of Theorem 5: Assuming that $I_{f}(2 t)<1$, we need to show that the Marchenko equation,

$$
\mathscr{K}_{t}(s)+\int_{t}^{\infty} \mathscr{K}_{t}(x) \mathscr{F}(s+x) d x=-\mathscr{F}(s+t),
$$

can be solved by the iteration defined in (50), and the solution satisfies the estimate in (51). The proof is a simple induction argument. Clearly $\left\|\mathscr{K}_{t}^{0}(s)\right\| \leq M_{f}(t+s) \leq$ $M_{f}(2 t)$. Assume that

$$
\left\|\mathscr{K}_{t}^{j}(s)\right\| \leq M_{f}(2 t)\left[1+I_{f}(2 t)+\ldots I_{f}^{j}(2 t)\right] .
$$

It follows from this assumption that

$$
\left\|\mathscr{F}_{t}^{j+1}(s)\right\| \leq M_{f}(t+s)+\left[1+I_{f}(2 t)+\ldots I_{f}^{j}(2 t)\right] \times \int_{t}^{\infty} M_{f}(2 t)\|\mathscr{F}(s+x)\| d x .
$$

As $s>t$, the induction hypothesis (70), with $j$ replaced by $j+1$ follows easily from this estimate. Using a similar argument we can show that, for $j \geq 1$,

$$
\left\|\mathscr{K}_{t}^{j}(s)-\mathscr{K}_{t}^{j-1}(s)\right\| \leq M_{f}(2 t) I_{f}(2 t)^{j}
$$

If $I_{f}(2 t)<1$, then this estimate shows that $\left\{\mathcal{K}_{t}^{j}\right\}$ is a uniformly convergent sequence for $s \in[t, \infty)$. The limit, $\mathscr{K}_{t}^{\infty}$, is evidently a solution to (69). We can also pass to the limit in (70) to conclude that $\mathscr{K}_{t}^{\infty}$ satisfies (51).

The proof of Theorem 6: The proof of this result is a small modification of the proof of the estimate (18) given in [1]. Let $\sigma$ denote the $2 \times 2$-matrix

$$
\sigma=\left[\begin{array}{cc}
-1 & 0 \\
0 & 1
\end{array}\right]
$$

and let

$$
D^{ \pm}(\xi ; t)=e^{-i t \xi \sigma}\left(F_{1}^{ \pm}-F_{2}^{ \pm}\right)
$$


For real $\xi$ and $t$, the matrices $e^{ \pm i t \xi \sigma}$ are unitary and therefore

$$
\left\|F_{1}^{ \pm}-F_{2}^{ \pm}\right\|=\left\|D^{ \pm}\right\| .
$$

The vector valued functions $D^{ \pm}$satisfy the equations

$$
\begin{aligned}
& \partial_{t} D^{ \pm}=e^{-i t \xi \sigma}\left[\begin{array}{cc}
0 & h_{1} \\
-h_{1}^{*} & 0
\end{array}\right] e^{i t \xi \sigma} D^{ \pm}+e^{-i t \xi \sigma}\left[\begin{array}{cc}
0 & \left(h_{1}-h_{2}\right) \\
-\left(h_{1}^{*}-h_{2}^{*}\right) & 0
\end{array}\right] F_{2}^{ \pm} . \\
& \lim _{t \rightarrow \pm \infty} D^{ \pm}(\xi ; t)=0 .
\end{aligned}
$$

We now restrict our attention to the - case, the + case is handled by an essentially identical argument.

The differential equation in (72) is equivalent to the integral equation

$$
\begin{aligned}
D^{-}(\xi ; t)= & \int_{-\infty}^{t} e^{-i s \xi \sigma}\left[\begin{array}{cc}
0 & h_{1}(s) \\
-h_{1}^{*}(s) & 0
\end{array}\right] e^{i s \xi \sigma} D^{-}(\xi ; s) d s+ \\
& \int_{-\infty}^{t} e^{-i s \xi \sigma}\left[\begin{array}{cc}
0 & \left(h_{1}-h_{2}\right)(s) \\
-\left(h_{1}^{*}-h_{2}^{*}\right)(s) & 0
\end{array}\right] F_{2}^{-}(s) d s .
\end{aligned}
$$

This is a Volterra equation which can be solved by the following iteration:

$$
\begin{aligned}
& D_{0}^{-}(\xi ; t)=\int_{-\infty}^{t} e^{-i s \xi \sigma}\left[\begin{array}{cc}
0 & \left(h_{1}-h_{2}\right)(s) \\
-\left(h_{1}^{*}-h_{2}^{*}\right)(s) & 0
\end{array}\right] F_{2}^{-}(s) d s \\
& D_{j}^{-}(\xi ; t)=D_{0}^{-}(\xi ; t)+\int_{-\infty}^{t} e^{-i s \xi \sigma}\left[\begin{array}{cc}
0 & h_{1}(s) \\
-h_{1}^{*}(s) & 0
\end{array}\right] e^{i s \xi \sigma} D_{j-1}^{-}(\xi ; s) d s,
\end{aligned}
$$

for $j \geq 1$.

First observe that the operator norm defined by the Euclidean norm satisfies

$$
\left\|\left[\begin{array}{cc}
0 & \alpha \\
-\alpha^{*} & 0
\end{array}\right]\right\|=|\alpha|,
$$

and therefore (18) implies that

$$
\left\|D_{0}^{-}(\xi ; t)\right\| \leq 2 I_{0}\left(\left\|h_{2}\right\|_{L^{1}}\right) H^{-}(t) .
$$

We make the inductive assumption that

$$
\left\|D_{j}^{-}(\xi ; t)\right\| \leq 2 I_{0}\left(\left\|h_{2}\right\|_{L^{1}}\right) H^{-}(t)\left[\sum_{k=0}^{j} \frac{\left(Q^{-}(t)\right)^{k}}{k !}\right] .
$$


From these assumptions and (74) it follows that

$$
\left\|D_{j+1}^{-}(\xi ; t)\right\| \leq 2 I_{0}\left(\left\|h_{2}\right\|_{L^{1}}\right)\left(H^{-}(t)+\int_{-\infty}^{t}\left|h_{1}(s)\right| H^{-}(s)\left[\sum_{k=0}^{j} \frac{\left(Q^{-}(s)\right)^{k}}{k !}\right]\right) .
$$

The definition of $Q^{-}$implies that

$$
\left|h_{1}(s)\right|=\partial_{s} Q^{-}(s),
$$

and therefore (76) implies that

$$
\begin{aligned}
\left\|D_{j+1}^{-}(\xi ; t)\right\| & \leq 2 I_{0}\left(\left\|h_{2}\right\|_{L^{1}}\right) H^{-}(t)\left[1+\int_{-\infty}^{t} \partial_{s}\left(\sum_{k=0}^{j} \frac{\left(Q^{-}(s)\right)^{k+1}}{(k+1) !}\right) d s\right] \\
& =2 I_{0}\left(\left\|h_{2}\right\|_{L^{1}}\right) H^{-}(t)\left(\sum_{k=0}^{j+1} \frac{\left(Q^{-}(s)\right)^{k}}{k !}\right) .
\end{aligned}
$$

Thiss completes the proof of the induction step. As

$$
\lim _{j \rightarrow \infty} D_{j}^{-}=D^{-}
$$

letting $j$ tend to infinity, completes the proof of the theorem.

\section{References}

[1] M. Ablowitz, D. Kaup, A. Newell, And H. Segur, The inverse scattering transform-Fourier analysis for nonlinear problems, Studies in Applied Math., 53 (1974), pp. 249-315.

[2] R. BEALS AND R. COIFMAN, Scattering and inverse scattering for first order systems, CPAM, 37 (1984), pp. 39-90.

[3] _ Scattering and inverse scattering for first order systems: II, Inverse Problems, 3 (1987), pp. 577-593.

[4] R. BeAls, P. Deift, And C. Tomei, Direct and inverse scattering on the line, American Mathematical Society, Providence, 1988.

[5] J. CARLSON, Exact solutions for selective-excitation pulses, Jour. of Mag. Res., 94 (1991), pp. 376-386.

[6] - Exact solutions for selective-excitation pulses. II. Excitation pulses with phase control, Jour. of Mag. Res., 97 (1992), pp. 65-78.

[7] I. DAUBECHIES, Ten lectures on wavelets, CBMS-NSF conference series in applied mathematics, SIAM, Philadelphia, 1992.

[8] L. Faddeev and L. Takhtajan, Hamiltonian Methods in the Theory of Solitons, Springer Verlag, Berlin, Heidelberg, New York, 1987.

[9] F. GRÜNBAUM AND A. HASENFELD, An exploration of the invertibility of the Bloch transform, Inverse Problems, 2 (1986), pp. 75-81. 
[10] P. LE Roux, Exact synthesis of radio frequency waveforms, in Proceeding of 7 th annual meeting of the SMRM, 1988, p. 1049.

[11] H. E. Moses AND R. T. Prosser, Eigenvalues and eigenfunctions associated with the Gel'fand-Levitan equation, J. Math. Phys., 25 (1984), pp. 108-112.

[12] L. P. PANYCH, Theoretical comparision of Fourier and wavelet encoding in magnetic resonance imaging, IEEE Trans. on Med. Imag., 15 (1996), pp. 141-153.

[13] J. Pauly, P. Le Roux, D. Nishimura, And A. Macovski, Parameter relations for the Shinnar-Le Roux selective excitation pulse design algorithm, IEEE Trans. on Med. Imaging, 10 (1991), pp. 53-65.

[14] D. E. ROURKE AND P. G. MORRIS, The inverse scattering transform and its use in the exact inversion of the Bloch equation for noninteracting spins, Jour. of Mag. Res., 99 (1992), pp. 118-138.

[15] W. Rudin, Real and Complex Analysis, McGraw Hill, New York, 1966.

[16] M. SHINNAR, L. BOLINGER, AND J. LEIGH, The synthesis of soft pulses with a specified frequency response, Mag. Res. in Med., 12 (1989), pp. 88-92.

[17] M. Shinnar, S. ElefF, H. Subramanian, AND J. Leigh, The synthesis of pulse sequences yielding arbitrary magnetization vectors, Mag. Res. in Med., 12 (1989), pp. 74-88.

[18] M. SHINNAR AND J. LEIGH, The application of spinors to pulse synthesis and analysis, Mag. Res. in Med., 12 (1989), pp. 93-98.

[19] E. M. STEIN AND G. WeISs, Introduction to Fourier Analysis on Euclidean Spaces, Princeton Press, Princeton, NJ, 1971.

[20] H. Widom, Lectures on Integral Equations, Van Nostrand; Reinhold Co., New YorkToronto, Ont.-London, 1969.

[21] V. Zakharov AND L. FAdDEEV, Korteweg-de Vries equation, a completely integrable Hamiltonian system, Funk. Anal. Prilŏz., 5 (1971), pp. 18-27.

[22] V. ZAKhaROV AND S. MANAKOV, On the complete integrability of the non-linear Schrödinger equation, Teor. Mat. Fiz., 19 (1974), pp. 332-343. 